\title{
NORMAL STRUCTURE OF THE ONE-POINT STABILIZER OF A DOUBLY-TRANSITIVE PERMUTATION GROUP. I
}

\author{
BY
}

MICHAEL E. O'NAN $\left({ }^{1}\right)$

\begin{abstract}
Let $G$ be a doubly-transitive permutation group on a finite set $X$ and $x$ a point of $X$. Let $N^{x}$ be a normal subgroup of $G_{x}$, the subgroup fixing $x$, such that $N^{x}$ is a T.I. set and not semiregular on $X-x$. Then, $\operatorname{PSL}(n, q) \subseteq G \subseteq P \Gamma L(n, q)$. Geometrical consequences of this result are also obtained.
\end{abstract}

Introduction. In this paper we obtain some general structure theorems about the one-point stabilizer of a doubly-transitive permutation group. If $G$ is doubly-transitive on $X$ and $x \in X$, we frequently denote a normal subgroup of $G_{x}$ by $N^{x}$. Then, for other $y$, we define $N^{y}$ so that $f N^{y} f^{-1}=N^{f(y)}$. In other words, $N^{y}$ is the unique conjugate of $N^{x}$ lying in $G_{y}$. Also, $N_{y}^{x}$ is the subgroup of $N^{x}$ fixing $y$, with $y \in X-x$.

In general we have $1 \subseteq N^{x} \cap N^{y} \subseteq N_{y}^{x}$, where all inclusions may be proper. In the known examples, however, it seems that if $N^{x}$ is intransitive on $X-x$, then either $N^{x} \cap N^{y}=1$ for $x \neq y$ or $N_{y}^{x}=N^{x} \cap N^{y}$ for $x \neq y$. The former is equivalent to the statement that $N^{x}$ is a T.I. set in $G$, the latter to the statement that $N^{x}$ is a strongly closed subgroup of $G_{x}$ in $G$.

In this paper we study the case in which $N^{x}$ is a T.I. set. Now if $N^{x}$ is semiregular on $X-x$, then $N^{x}$ is clearly a T.I. set. However, this case seems to be better treated by other means than those we employ herein. Accordingly, in addition, we assume $N_{y}^{x} \neq 1$, i.e. $N^{x}$ is not semiregular on $X-x$. We then prove

TheOREM A. Let $G$ be a doubly-transitive group on a finite set $X$ and $N^{x}$ a normal subgroup of $G_{x}$. Assume that $N^{x}$ is a T.I. set in $G$ and $N^{x}$ is not semiregular on $X-x$. Then $\operatorname{PSL}(n, q) \subseteq G \subseteq P \Gamma L(n, q), n \geqslant 3$.

The following is an immediate consequence of Theorem A. Block designs are defined in $\S 1$. Usually they are called balanced incomplete block designs with $\lambda=1$.

Presented to the Society, January 26, 1973; received by the editors May 6, 1974. AMS (MOS) subject classifications (1970). Primary 20B20; Secondary 20B10, $20 \mathrm{~B} 25$.

(1) During the preparation of this research the author was partially supported by the National Science Foundation under Grant GP-21071. 
THEOREM B. Let $B$ be a proper block design on a finite set $X$ and suppose $\operatorname{Aut}(B)$ is doubly-transitive. Assume there is an element $f \neq 1, f \in \operatorname{Aut}(B)$, such that

(i) f fixes $x \in X$ and all blocks which contain $x$, and

(ii) $f$ fixes a point $y \in X, y \neq x$.

Then $B$ is a projective geometry over a finite field.

In the course of proving Theorem A, we obtain

THEOREM C. Let $G$ be a doubly-transitive group on $X$ and $N^{x}$ a normal subgroup of $G_{x}$. Assume $\left|N^{x}\right|$ is even and if $y \in X-x,\left|N_{y}^{x}\right|$ is odd. Then, if $j$ is an involution in $N^{x}$,

(i) $N^{x}=C_{N^{x}}(j) N_{y}^{x}$, and

(ii) if $N_{y}^{x}$ is nilpotent, $O_{2}\left(N^{x}\right) \neq 1$.

In the latter case, the structure of $G$ follows from a theorem of Shult [20]. We also note that Hering [19] has obtained a similar result.

The bulk of the work in this paper is connected with proving Theorem A. We note that in $\operatorname{PGL}(n, q), n \geqslant 3$, if we take $A^{x}$ to be the subgroup consisting of the identity together with transvections with center $x, A^{x}$ satisfies the conditions of Theorem A. Moreover, in $\operatorname{PGL}(n, q)_{x}$, there is a normal subgroup $N^{x}$ of order $q^{n-1}(q-1)$, containing $A^{x}$ and satisfying the conditions of Theorem A. Also, in the groups $\operatorname{PSL}(3,2)$ and $\operatorname{PSL}(3,3)$, there are subgroups not of the above type which satisfy the conditions of Theorem A. Our main objective is to recover the structure of the subgroups and obtain the hypotheses of Theorem $A$ of [11].

$\S 1$ consists mostly of general results. In $\S 2$, we obtain a proof of Theorem $\mathrm{C}$. The proof is inductive, relying heavily on the technique of building smaller block designs in which the hypotheses of the theorem hold.

In $\S 3$ we begin the proof of Theorem A. The main fact upon which most of the subsequent analysis rests is Lemma 3.3, which asserts that $N_{x}^{y}$ normalizes $N^{x}$ and for all $f \in N_{x}^{y}, f \neq 1, C_{N^{x}}(f)=N_{y}^{x}$. In the first half of this section, we draw some geometric consequences of this lemma.

In the second half of $\S 3$, we eliminate the possibility that $N^{x}$ is a Frobenius group having Frobenius complement $N_{y}^{x}$. The proof of this is largely geometrical. At the end of $\S 3$ (Lemma 3.19), we state our major tool for identifying $\operatorname{PSL}(n, q)$.

In $\S 4$ we begin the structural analysis of $N^{x}$. This is done by considering triples of groups $(H, K, L)$, where $L$ is a proper subgroup of $K$ and $H$ is a subgroup of $\operatorname{Aut}(K)$ having the property that if $\varphi \in H, \varphi \neq 1, C_{K}(\varphi)=L$. We call this triple an $(H, K, L)$ configuration. By Lemma 3.3, with $H=N_{x}^{y}, K=N^{x}$, 
and $L=N_{y}^{x}$, we have an $\left(N_{x}^{y}, N^{x}, N_{y}^{x}\right)$ configuration. By the fact that $G$ is doubly-transitive, $N_{y}^{x} \cong N_{x}^{y}$, and so $H \cong L$. We make use of this side condition.

The main result of $\S 4$ (Proposition 4.9) asserts that in an $(H, K, L)$ configuration with $H \cong L$ we have either

(1) $H$ is a Frobenius complement, or

(2) $H$ is an abelian group, or

(3) both $H$ and $K$ are Frobenius groups and $L$ intersects the center of the Frobenius kernel of $K$ in a nontrivial manner.

This result dictates the lines of most of our subsequent analysis. In case (3), if $H=N_{x}^{y}$ and $K=N^{x}$, it follows that $G_{x}$ has an abelian normal subgroup which is not semiregular on $X-x$, and by Theorem A of [11], our Theorem A follows. Thus we restrict our attention to cases (1) and (2).

In case (1), we obtain (Proposition 4.15), in an $(H, K, L)$ configuration with $H \cong L$ and $H$ a nonabelian Frobenius complement, that $L$ is a Hall subgroup of $K$ and has a normal complement in $K$. Again, using Lemma 3.19, this result is sufficient to obtain the conclusion of Theorem A.

When $H=N_{x}^{y}$ is abelian of odd order, or has cyclic Sylow 2-subgroup, as we see in $\S 5$, the conclusion of Theorem A can be obtained directly. Accordingly, in the remainder of $\S 4$, we assume that the Sylow 2-subgroup of $H$ is noncyclic and that $H$ is abelian. We impose the additional side condition that $\left[H, N_{K}(L)\right] \subseteq L$ (which we know to be valid when $H=N_{x}^{y}, K=N^{x}$, and $L=$ $N_{y}^{x}$, by Lemma 3.5) and analyze the structure of $K$. It turns out (Proposition 4.26) that either $L \triangleleft K$ or $K \cong S L\left(2,2^{\alpha}\right)$ and $H$ and $L$ are elementary abelian of order $2^{\alpha}$.

Using this specific structural information, in $\$ 5$ we complete the proof of Theorem A.

1. Generalities. In this section we consider some general definitions and properties of permutation groups and block designs. The notations and lemmas are mostly those of [11]. We review these briefly. Proofs are found in [11].

In the following all groups and sets are finite.

If $G$ is a permutation group on a set $X$ and $H \subseteq G$, by $F_{H}$ we mean the subset of $X$ whose points are fixed by all elements of $H$. If $B \subseteq X, G_{B}$ is the subgroup of $G$ which fixes all points of $B$ and $G_{B}^{*}$, the subgroup of $G$ which fixes the set $B$. If $K \subseteq G_{B}^{*}, K \mid B$ is the permutation group obtained by restricting $K$ to $B$. $G$ is semiregular on $X$ if only $1 \in G$ fixes points of $X$.

A block design $B$ on a set $X$ is a family of subsets $B$ of $X$ with the properties:

(i) If $B_{1}, B_{2} \in B,\left|B_{1}\right|=\left|B_{2}\right|$.

(ii) Every two element subset of $X$ belongs to a unique member of $B$. (This would usually be called a block design with $\lambda=1$.) If $a, b \in X, B(a, b)$ 
is the unique block of $B$ which contains $a$ and $b . B_{a}$ is the family of blocks containing $a$. A block design is proper if $2<|B|<|X|$ for $B \in B$. Automorphisms of block designs are defined in the usual way.

Lemma 1.1. Let $B$ be a proper block design on $X$. Suppose $|X|=1+n$ and $|B|=1+s$ if $B \in B$. Then:

(i) There are $(1+n) n /(1+s) s$ blocks in $B$.

(ii) Every point of $B$ lies in $n / s$ blocks.

(iii) $n \geqslant s+s^{2}$.

If $B$ is a block design on $X$ and $Y \subseteq X$, we say that $Y$ is a subspace over $B$ if whenever $B \in B$ and $|B \cap Y| \geqslant 2$, then $B \subseteq Y$.

LEMMA 1.2. If $Y_{1}$ and $Y_{2}$ are subspaces over $B$, so is $Y_{1} \cap Y_{2}$.

If $X$ is a set, $\delta(X)=\{(x, x) \mid x \in X\} \subseteq X \times X$.

If $G$ is a doubly-transitive group on a set $X$, a predesign function for $G$ is a function $\Delta$ which assigns to each $(x, y) \in X \times X-\delta(X)$, a subset $\Delta(x, y)$ of $X$, having the properties:

(i) $\{x, y\} \subseteq \Delta(x, y)$.

(ii) If $z \in \Delta(x, y)-x, \Delta(x, y)=\Delta(x, z)$.

(iii) $g(\Delta(x, y))=\Delta(g(x), g(y))$, if $g \in G$.

Then we have $[11, \S 1]$

LEMMA 1.3. There is a one-one correspondence of predesign functions for $G$ and systems of imprimitivity of $G_{x}$ on $X-x$.

LEMmA 1.4. Let $G$ be a doubly-transitive group on a set $X$ and $\Delta$ be a predesign function for $G$. Then one of the following holds:

(i) If $\Delta(x, y)=\Delta(y, x)$ for some $(x, y) \in X \times X-\delta(X)$, then $\Delta(x, y)=$ $\Delta(y, x)$, for all $(x, y) \in X \times X-\delta(X)$, and the family of sets $\{\Delta(x, y)\}$ forms a block design on $X$ preserved by $G$.

(ii) If $\Delta(x, y) \neq \Delta(y, x)$, then whenever $\Delta(a, b)=\Delta(c, d)$, we have $a=c$ and $d \in \Delta(a, b)-a$.

LEMMA 1.5. Suppose $\Delta$ is a predesign function for a doubly-transitive group $G$ on a set $X$, in which $\Delta(x, y) \neq \Delta(y, x)$. Suppose $|X|=1+n$ and $|\Delta(x, y)|=1+$ s. Then:

(i) There are $(1+n) n / s$ distinct sets $\Delta(x, y)$.

(ii) Every pair of points of $X$ is contained in exactly $1+s$ sets $\Delta(x, y)$.

If $G$ is a doubly-transitive group on a set $X$ and $N^{x}$ is a normal subgroup of $G_{x}$, we define $N^{y}$ for other $y \in X$ so that $f N^{y} f^{-1}=N^{f(y)}$, for $f \in G$. There are two predesign functions associated with $N^{x}$ which will be of particular importance in what follows. 
LEMMA 1.6. The functions

$$
\Delta_{N}(x, y)=x \cup\left\{f(y) \mid f \in N^{x}\right\} \text { and } \Gamma_{N}(x, y)=F_{N_{y}^{x}}
$$

are predesign functions for $G$ on $X[11, \S 2]$.

LEMma 1.7. $\Gamma_{N}(x, y)=\Gamma_{N}(y, x)$ and the family of sets $\left\{\Gamma_{N}(x, y)\right\}$ forms a block design on $X$ preserved by $G[11$, Corollary $\mathrm{B} 1]$.

More generally, suppose $K$ is a strongly closed subgroup of $G_{x y}$ in $G_{x}$. Then $K \triangleleft G_{x y}$ and we may define $K(u, v)$ for other $(u, v) \in X \times X-\delta(X)$, so that $g K(u, v) g^{-1}=K(g(u), g(v))$. Then we have

LEMma 1.8. The function $\Gamma_{K}(x, y)=F_{K(x, y)}$ is a predesign function for $G$ on $X$ and the family of sets $\left\{\Gamma_{K}(x, y)\right\}$ forms a block design on $X$ preserved by $G[11$, Theorem $\mathrm{B}]$.

Lemma 1.9. If a Sylow p-subgroup of $K(y, x)$ fixes $z \in X$, then some Sylow p-subgroup of $K(x, y)$ fixes $z[11$, Lemma 2.6] .

Lemmas 1.7 and 1.8 resemble a result of Witt [18].

LEMMA 1.10. If $W$ is a weakly closed subgroup of $G_{x y}$ in $G$, then the family of sets $\left\{F_{c W_{c}}{ }^{-1} \mid c \in G\right\}$ forms a block design on $X$, preserved by $G$.

Actually, Lemma 1.8 can be used to construct such a weakly closed subgroup.

Lemma 1.11. Suppose, as in Lemma 1.8, that $K(x, y)$ is a strongly closed subgroup of $G_{x y}$ in $G_{x}$. Set $B=\Gamma_{K}(x, y)$. Then: $G_{x y}$ in $G$.

(i) $W(x, y)=\Pi_{(u, v) \in B \times B-\delta(B)} K(u, v)$ is a weakly closed subgroup of

(ii) If $(u, v) \in B \times B-\delta(B), K(u, v) \triangleleft W(x, y)$.

(iii) $F_{W(x, y)}=B$.

Proof. We show that $W(x, y)$ is the weak closure of $K(x, y)$ in $G_{x y}$. Now a conjugate $K(u, v)$ of $K(x, y)$ is contained in $G_{x y}$ if and only if $\{x, y\}$ $\subseteq F_{K(u, v)}=\Gamma_{K}(u, v)$. But by Lemma $1.8,\{x, y\} \subseteq \Gamma_{K}(u, v)$ if and only if $\Gamma_{K}(x, y)=\Gamma_{K}(u, v)=B$. Thus, $K(u, v) \subseteq G_{x y}$ if and only if $(u, v) \in B \times B-$ $\delta(B)$.

Thus, $W(x, y)=\langle K(u, v) \mid(u, v) \in B \times B-\delta(B)\rangle$ is the weak closure of $K(x, y)$ in $G_{x y}$, and $F_{W(x, y)}=\Gamma_{K}(x, y)=B$.

Now if $(s, t) \in B \times B-\delta(B), K(s, t) \triangleleft G_{s t}$ and $W(x, y) \subseteq G_{B} \subseteq G_{s t}$, and so $K(s, t) \triangleleft W(x, y)$. Thus, $W(x, y)=\Pi_{(u, v) \in B \times B-\delta(B)} K(u, v)$.

REMARK. With $B=\Gamma_{K}(x, y)$ as in Lemma 1.11 , if $(u, v) \in B \times B-$ $\delta(B)$ and $\left(u, v^{\prime}\right) \in B \times B-\delta(B)$, then $K(u, v)=K\left(u, v^{\prime}\right)$. Thus, the product 
for $W(x, y)$ need be taken only over $|B|$ groups $K(u, v)$, one for each $u \in B$. Of use will be

LEMMA 1.12. $N_{N^{x}}\left(N_{y}^{x} N_{x}^{y}\right)=N_{N^{x}}\left(N_{y}^{x}\right)[11$, Corollary B2]

More generally, we have

LEMmA 1.13. Suppose $K(x, y) \subseteq N_{y}^{x}$ and $K(x, y)$ is a strongly closed subgroup of $G_{x y}$ in $G_{x}$. Suppose also that

(i) $K(x, y) K(y, x)$ is a weakly closed subgroup of $N_{y}^{x} K(y, x)$ in $N^{x} K(y, x)$, and

(ii) $K(y, x) \cap N^{x} \subseteq K(x, y)$.

Then $N_{N^{x}}(K(x, y) K(y, x))=N_{N^{x}}(K(x, y))$.

PROOF. Of course, $K(x, y) \triangleleft G_{x y}, K(y, x) \triangleleft G_{x y}$, and $N_{y}^{x} \triangleleft G_{x y}$. Also since $K(y, x)$ fixes $x, K(y, x)$ normalizes $N^{x}$. Thus, $K(x, y) K(y, x), N_{y}^{x} K(y, x)$, and $N^{x} K(y, x)$ are all groups. Also, since $K(y, x) \subseteq G_{x y}, K(y, x)$ fixes the set $\Delta_{N}(x, y)-x$.

Now the groups $N^{x}$ and $N^{x} K(y, x)$ are both transitive on the set $\Delta_{N}(x, y)-x=Y$. We compare their action on $Y$. In the first group the stabilizer of the point $y \in Y$ is $N_{y}^{x}$ and in the second the stabilizer of $y$ is $N_{y}^{x} K(y, x)$. Now, since $K(x, y)$ is a strongly closed subgroup of $G_{x y}$ in $G_{x}, K(x, y)$ is a weakly closed subgroup of $N_{y}^{x}$ in $N^{x}$. By Witt's theorem [18], $N_{N^{x}}(K(x, y))$ is transitive on $F_{K(x, y)} \cap Y=\Gamma_{K}(x, y) \cap Y$. By hypothesis, $K(x, y) K(y, x)$ is a weakly closed subgroup of $N_{y}^{x} K(y, x)$ in $N^{x} K(y, x)$. Again by Witt's theorem, $N_{N} \dot{x}_{K(y, x)}(K(x, y) K(y, x))$ is transitive on $F_{K(x, y)} \cap F_{K(y, x)} \cap Y=\Gamma_{K}(x, y)$ $\cap \Gamma_{K}(y, x) \cap Y$. Clearly,

$$
N_{N^{x} K(y, x)}(K(x, y) K(y, x))=K(y, x) N_{N^{x}}(K(x, y) K(y, x)) .
$$

Thus, $N_{N^{x}}(K(x, y) K(y, x))$ is transitive on $\Gamma_{K}(x, y) \cap \Gamma_{K}(y, x) \cap Y$. Also, by Lemma 1.8 (since $K(x, y)$ is a strongly closed subgroup of $G_{x y}$ in $\left.G_{x}\right), \Gamma_{K}(x, y)$ $=\Gamma_{K}(y, x)$. Therefore, $N_{N} x(K(x, y) K(y, x))$ is transitive on $\Gamma_{K}(x, y) \cap Y$.

Next, note that $N_{N^{x}}(K(x, y) K(y, x))$ normalizes $N^{x} \cap(K(x, y) K(y, x))=$ $K(x, y)\left(N^{x} \cap K(y, x)\right)=K(x, y) \quad$ (using hypothesis (ii)). Therefore, $N_{N^{x}}(K(x, y) K(y, x)) \subseteq N_{N^{x}}(K(x, y))$.

Since $K(x, y) \triangleleft G_{x y}$ and $K(y, x) \triangleleft G_{x y}, N_{y}^{x} \subseteq N_{N^{x}}(K(x, y) K(y, x))$ and $N_{y}^{x} \subseteq N_{N^{x}}(K(x, y))$. Thus the stabilizer of $y$ in $N_{N^{x}}(K(y, x) K(x, y))$ is $N_{y}^{x}$ and the stabilizer of $y$ in $N_{N^{x}}(K(x, y))$ is $N_{y}^{x}$.

It follows that $N_{N^{x}}(K(x, y) K(y, x)) \subseteq N_{N^{x}}(K(x, y))$, both groups are transitive on the set $Y \cap \Gamma_{K}(x, y)$, and in both groups, the stabilizer of $y \in Y$ is $N_{y}^{x}$. Therefore, $N_{N^{x}}(K(x, y) K(y, x))=N_{N^{x}}(K(x, y))$.

One case under which the hypotheses of Lemma 1.13 are readily verified 
LEMMA 1.14. Suppose $N_{y}^{x}$ is p-closed and take $P(x, y)$ to be the normal Sylow p-subgroup of $N_{y}^{x}$. Then, $N_{N^{x}}(P(x, y) P(y, x))=N_{N^{x}}(P(x, y))$.

An interpretation of Lemma 1.13 comes from

LEMMA 1.15. Under the hypotheses of Lemma $1.13, K(y, x)$ normalizes $N_{N^{x}}(K(x, y))$ and acts trivially on $N_{N^{x}}(K(x, y)) / K(x, y)$.

Proof. $K(y, x)$ normalizes $K(x, y)$ and $N^{x}$, and so, $N_{N^{x}}(K(x, y))$. Thus, $\left[N_{N^{x}}(K(x, y)), K(y, x)\right] \subseteq N^{x}$. Also, by Lemma 1.13, [ $\left.N_{N^{x}}(K(x, y)), K(y, x)\right]$ $\subseteq K(x, y) K(y, x)$. Thus, $\left[N_{N^{x}}(K(x, y)), K(y, x)\right] \subseteq N^{x} \cap(K(x, y) K(y, x))=$ $K(x, y)$.

We say that a block design $B$ preserved by $G$ is $N$-admissible if $N^{x}$ fixes all blocks of $B_{x}$.

LEMMA 1.16. If $G$ preserves a propèr $N$-admissible block design $B$, then $N^{x} \cap N^{y}=1$, if $x \neq y$; i.e., $N^{x}$ is a T.I. set [11, Lemma 2.8].

LEMMA 1.17. Either

(i) $C\left(N^{x}\right)$ is semiregular on $X-x$, or

(ii) $G$ preserves a proper $N$-admissible block design [11, Corollary B3].

2. Theorem C. In this section $G$ is a doubly-transitive group on a set $X$ and $N^{x}$ is a normal subgroup of $G_{x}$. We assume that $\left|N^{x}\right|$ is even and $\left|N_{y}^{x}\right|$ is odd if $y \in X-x$. Thus, $N^{x}$ has involutions and each fixes only $x$.

We define $N^{y}$ for other $y$ so that $f N^{y} f^{-1}=N^{f(y)}$, for $f \in G$.

We begin by proving Theorem $\mathrm{C}(\mathrm{i})$, namely, if $j$ is an involution of $N^{x}$, then $N^{x}=C_{N^{x}}(j) N_{y}^{x}$, if $y \in X-x$.

We take $|X|=1+n$. Henceforth, $j$ will be a fixed involution of $N^{x}$ which interchanges $y$ and $z$ of $X-x$. Then $j$ normalizes $G_{y z}$, and since $j$ fixes only $x$, $\left\langle G_{y z}, j\right\rangle$ has a unique orbit $\Lambda(y, z)$ of odd length. We let $|\Lambda(y, z)|=s$. Also, $j$ normalizes $N_{y z}^{x}$. We set $t=\left|N_{y z}^{x}: C_{N_{y z}^{x}}(j)\right|$. Since $\left|N_{y z}^{x}\right|$ is odd, $t$ is odd.

LEMMA 2.1. If $k$ is an invblution of $N^{y}, y \neq x$, then $|\cdot k|$ is odd.

Proof. $\langle j, k\rangle$ is a dihedral group having a unique orbit $\Delta$ of odd length, since $j$ fixes a single point. Since $j$ fixes $x \in \Delta$ and $k$ fixes $y \in \Delta,|\Delta| \geqslant 3$.

Let $b$ generate the subgroup of $\langle j k\rangle$ which fixes all points of $\Delta$. Set $i=b j$. Then $i \in\langle j, k\rangle-\langle j k\rangle$ and $i$ is an involution. Since all involutions of $\langle j, k\rangle-\langle j k\rangle$ are conjugate to either $j$ or $k, i$ is conjugate to some involution of $N^{x}$. Since $j|\Delta=i| \Delta, i$ fixes $x$ and so $i \in N^{x}$. Thus, $b=i j \in N^{x}$. Since $b$ fixes $y \in \Delta$, $y \neq x, b \in N_{y}^{x}$ and $|b|$ is odd. Since $|j k|=|\Delta||b|,|j k|$ is odd.

Lemma 2.2. All involutions of $N^{x}$ are conjugate in $G$, hence in $G_{x}$. 
LEMMA 2.3. If $k$ is an involution of $N^{x}$ interchanging $y$ and $z,|j k|$ is odd.

Proof. $j k \in N_{y}^{x}$ and $\left|N_{y}^{x}\right|$ is odd.

LEMMA 2.4. (i) If $k$ is an involution of $N^{x^{\prime}}$ which interchanges $y$ and $z, j$ and $k$ are conjugate in $\left\langle G_{y z}, j\right\rangle$.

(ii) $G_{y z}=O\left(G_{y z}\right) C_{G_{y z}}(j)$.

(iii) $j$ has st conjugates in $\left\langle G_{y z}, j\right\rangle$, i.e., st conjugates of $j$ interchange $y$ and $z$.

(iv) If $j^{\prime}$ is an involution of $N^{x^{\prime}}$ which interchanges $y^{\prime}$ and $z^{\prime}$, there is an $f \in G$, such that $f^{-1} j^{\prime} f=j, f(x)=x^{\prime}, f(y)=y^{\prime}$, and $f(z)=z^{\prime}$. In particular, $N_{y z}^{x}$ and $N_{y^{\prime} z^{\prime}}^{x^{\prime}}$ are conjugates, $C_{N_{y z}^{x}}(j)$ and $\left.C_{N_{y}^{\prime} z^{\prime}}^{x^{\prime}}, j^{\prime}\right)$ are conjugates, and $\left|N_{y z}^{x}: C_{N_{y z}^{x}}(j)\right|=t$.

(v) $j$ has $(1+n)$ st conjugates in $G$, st conguates in $G_{x}$.

(vi) $C_{G}(j)$ is transitive on $X-x$.

(vii) $j$ is central in some Sylow 2-subgroup of $N^{x}$.

Proof. (i) and (ii). If $x \neq x^{\prime}$, by Lemma $2.1, j k$ is of odd order. If $x=x^{\prime}$, by Lemma $2.3, j k$ is of odd order. Thus, $j$ and $k$ are conjugate in $\left\langle G_{y z}, j\right\rangle$ and $j$ has a unique conjugate in any Sylow 2-subgroup of $\left\langle G_{y z}, j\right\rangle$. It follows that $j$ is isolated and from Glauberman's $Z^{*}$-theorem [8], $G_{y z}=$ $O\left(G_{y z}\right) C_{G_{y z}}(j)$, and (i) and (ii) follow.

(iii) Let $r$ be the number of conjugates of $j$ in $\left\langle G_{y z}, j\right\rangle$ which fix $x$. Then $j$ has $s r$ conjugates in $\left\langle G_{y z}, j\right\rangle$. Any conjugate of $j$ in $\left\langle G_{y z}, j\right\rangle$ which fixes $x$ belongs to $\left\langle N_{y z}^{x}, j\right\rangle$, the subgroup of $N^{x}$ which fixes the set $\{y, z\}$. Since $\left|N_{y}^{x}\right|$ is odd, $r=\left|N_{y z}^{x}: C_{N_{y z}^{x}}^{x}(j)\right|=t$.

(iv) Since $G$ is doubly-transitive on $X$, there is a $g \in G$ such that $g(y)=y^{\prime}$, $g(z)=z^{\prime}$. Then $g^{-1} j^{\prime} g$ interchanges $y$ and $z$. By (i), there is an $h \in G_{y z}$ such that $h^{-1} g^{-1} j^{\prime} g h=j$. Then, $(g h)(y)=y^{\prime},(g h)(z)=z^{\prime}$, and $(g h)(x)=x^{\prime}$. Setting $f=g h,(v)$ follows.

(v) We count in two ways, the pairs $(k,\{a, b\})$, where $k$ is an involution of $N^{c}$ interchanging $a$ and $b$. If $\gamma$ is the number of conjugates of $j$, this number is $(m) / 2$. Now $X$ has $(1+n) n / 2$ two element subsets and by (iii) each two element subset is fixed by st conjugates of $j$, this number is also $((1+n) n / 2)(s t)$. Thus, $\gamma=(1+n)$ st.

(vi) We consider $G$ as a transitive permutation group on $X^{*}$, the family of two element subsets of $X$. Then $G_{\{y, z\}}^{*}$ is the subgroup of $G$ fixing $\{y, z\} \in$ $X^{*}$. If $j$ and $k$, a conjugate of $j$, fix $\{y, z\}$, by (i), $j$ and $k$ are conjugate in $G_{\{y, z\}}^{*}$. By Witt's theorem, $C_{G}(j)$ is transitive on the two element subsets of $X$ fixed by $j$. As $j$ is itself transitive on each such two element subset, $C_{G}(j)$ is transitive on $X-x$. 
(vii) $(1+n) s t$ is odd.

LeMma 2.5. Let $p$ be an odd prime divisor of $\left|G_{y z}\right|$. Then $j$ normalizes some Sylow p-subgroup $P$ of $G_{y z}$.

Thus any conjugate of $P$ is normalized by some conjugate of $j$.

Proof. Take $Q$ a Sylow $p$-subgroup of $C_{G_{y z}}(j)$. Then $Q O\left(G_{y z}\right)$ contains a Sylow $p$-subgroup of $G_{y z}$ and $j$ normalizes $Q O\left(G_{y z}\right)$. Since $\left|Q O\left(G_{y z}\right)\right|$ is odd, $j$ normalizes a Sylow $p$-subgroup of $Q O\left(G_{y z}\right)$, and so also one of $G_{y z}$.

LEMMA 2.6. Let $p$ be an odd prime and $P$ a Sylow p-subgroup of $G_{y z}$. If $\left|F_{p}\right|$ is even, $\left|F_{p}\right|=2$.

Proof. By Lemma 2.5, we may assume $j$ normalizes $P$. Set $\Delta=F_{p}$. By Witt's theorem, $N_{G}(P) \mid \Delta$ is doubly-transitive.

We claim: $j \mid \Delta$ is an isolated involution of $N_{G}(P) \mid \Delta$.

Suppose not. Then, $j$ has some conjugate $k$ in $N_{G}(P)$ such that $\langle j, k\rangle \mid \Delta$ is a four group. Then, since $\langle j, k\rangle$ is dihedral, there is some involution $i \in\langle j, k\rangle$, such that $i$ is conjugate to $j$ and $\langle j, i\rangle$ is a four group. Since $i j=j i, i$ fixes $x$ and so $i \in N^{x}$. Thus, $\langle i, j\rangle \subseteq N^{x}$.

Thus $\langle j, i\rangle \subseteq N_{G}(P)$ and $P$ is of odd order. It follows then that $P=$ $C_{P}(j) C_{P}(i) C_{P}(i j)$. Since $i, j$, and $i j$ fix only $x, C_{P}(j), C_{P}(i), C_{P}(i j) \subseteq G_{x}$. Thus, $P \subseteq G_{x}$. Thus, $x \in \Delta$. Since $j$ fixes $\Delta,|\Delta|$ is odd, contrary to hypothesis.

Therefore, $j \mid \Delta$ is an isolated involution of the doubly-transitive group $H=N_{G}(P) \mid \Delta$. By Glauberman's $Z^{*}$-theorem, $H=O(H) C_{H}(j)$. Since $H$ is doublytransitive on $\Delta$ and $|\Delta|$ is even, $O(H)=1$. Thus, $j \mid \Delta \in Z(H)$. Thus, $|\Delta|=2$.

The same proof yields

LEMMA 2.7. Let $W$ be a weakly closed subgroup of odd order of $G_{y z}$ in G. If $\left|F_{W}\right|$ is even, $\left|F_{W}\right|=2$.

Lemma 2.8. Let $p$ be a prime dividing s. Let $P$ be a Sylow p-subgroup of $G_{y z}$, then $\left|F_{p}\right|=2$, and $n \equiv 1(\bmod p)$.

Proof. Since $G_{y z}$ has an orbit of length $s, p \neq 1$. By Lemma 2.6 it suffices to show that $\left|F_{P}\right|$ is even.

Suppose $\left|F_{P}\right|$ is odd and take $j$ normalizing $P$, as we may by Lemma 2.5 , Since $F_{P}$ is odd and fixed by $j, x \in F_{P}$. Thus, $P$ fixes $x \in \Lambda(y, z)$.

But $G_{y z}$ is transitive on $\Lambda(y, z)$, and so all orbits of its Sylow $p$-subgroups will be of length divisible by $p[16, \mathrm{p} .6]$. Thus, $P$ can fix no point of $\Lambda(y, z)$, and $\left|F_{P}\right|$ is even.

Since $|X|=1+n$, it follows that $p \mid(n-1)$. 
Lemma 2.9. Let $p$ be a prime dividing s. Then $N_{y}^{x}$ contains a Sylow p-subgroup of $N^{x}$.

Proof. Take $R$ a Sylow $p$-subgroup of $N^{x}$. By Lemma 2.8, $R$ fixes some $y \in X-x$ and so $R \subseteq N_{y}^{x}$. Since all $N_{y}^{x}$ are conjugate under $G_{x}$ and $N^{x} \triangleleft G_{x}$, each $N_{y}^{x}$ contains a Sylow $p$-subgroup of $N^{x}$.

THEOREM C (i). $N^{x}=N_{y}^{x} C_{N}(j)$.

Proof. We show that $N_{y}^{x}$ acts transitively by conjugation on $C$, the class of conjugates of $j$ under $N^{x}$. Let $m=|C|$. Let $l$ be the number of $N_{y}^{x}$ conjugates of $j$. Then $l \leqslant m$.

Since $N^{x} \triangleleft G_{x}$ and $G_{x}$ acts transitively on the st involutions of $N^{x}, m \mid s t$.

Let $p^{\alpha}$ be the highest power dividing $m$ of some prime divisor $p$ of $m$.

If $p \mid s$, take $P$ a Sylow $p$-subgroup of $N_{y}^{x}$. By Lemma 2.9, $P$ is a Sylow $p$-subgroup of $N^{x}$. Thus, all orbits of $P$ on $C$ are of length divisible by $p^{\alpha}$ $\left[16\right.$, p. 6]. Therefore, $p^{\alpha} \mid l$.

If $p \nmid s$, then $p \mid t$ and $t=\left|N_{y z}^{x}: C_{N_{y z}^{x}}(j)\right|$. Since $j$ normalizes $N_{y z}^{x}$ of odd order, there is a Sylow $p$-subgroup $R$ of $N_{y z}^{x}$ normalized by $j$ and containing a Sylow $p$-subgroup of $C_{N_{y z}^{x}}(j)$. Then $p^{\alpha}=\left|R: C_{R}(j)\right|$.

We claim: all orbits of $R$ on $C$ are of length divisible by $p^{\alpha}$.

If this is not the case, there is some $k \in C$, such that $\left|R: C_{R}(k)\right|=p^{\beta}$ and $\beta<\alpha$. Set $Q=C_{R}(k)$ and $z^{\prime}=k(y)$. Then $Q \subseteq N_{y z^{\prime}}^{x}$ and $Q \subseteq C_{N_{y z^{\prime}}}(k)$. But since $C_{N_{y z}^{x}}(j)$ and $C_{N_{y z}^{x}}(k)$ are conjugate by Lemma 2.4(iv), this is impossible, as their Sylow subgroups are of order $|R| / p^{\alpha}$ and $p^{\gamma} \geqslant|R| / p^{\beta}>|R| / p^{\alpha}$, respectively.

It follows that $p^{\alpha} \mid l$, since $R$ is contained in some Sylow $p$-subgroup of $N_{y}^{x}$.

Therefore, $m \mid l$ and since $l \leqslant m, l=m$.

Corollary C1. If $N^{x}$ is semiregular and $X-x$, all involutions of $N^{x}$ are contained in $Z\left(N^{x}\right)$.

We shall show next that if $N_{y}^{x}$ is nilpotent, $O_{2}\left(N^{x}\right) \neq 1$. In the remainder of this section we assume then, in addition to our other hypotheses, that $N_{y}^{x}$ is nilpotent. We prove $O_{2}\left(N^{x}\right) \neq 1$ by induction on $|X|$. We begin with a preliminary lemma.

Lemma 2.10. Let $B$ be a block design on $X$ and suppose if $B \in B,|B|$ is odd. Suppose $j$ is an involution of $\operatorname{Aut}(B)$ fixing a single point $x$ of $X$. Then $j$ fixes all blocks of $B$ whcih contain $x$.

Proof. Let $B_{1}, B_{2}, \ldots, B_{l}$ be the blocks of $B$ which are fixed by $j$. Since $\left|B_{i}\right|$ is odd and $j$ fixes only $x, x \in B_{i}$, for all $i$. Since $B$ is a block design, $B_{i} \cap B_{j}=\{x\}$, if $i \neq j$. 
Now if $y \in X-x, j$ fixes the block $B(y, j(y))$, as $j$ fixes the set $\{y, j(y)\}$. Thus, $y$ belongs to some $B_{i}$. It follows that $X=\bigcup_{B_{i}}$. Thus, $B_{1}, \ldots, B_{l}$ exhausts $B_{x}$ and the lemma follows.

LemMA 2.11. $O_{2}\left(N^{x}\right) \neq 1$ if and only if all involutions of $N^{x}$ commute with each other.

PROOF. Clearly if all involutions of $N^{x}$ commute, $O_{2}\left(N^{x}\right) \neq 1$. So suppose $O_{2}\left(N^{x}\right) \neq 1$. Then $Z\left(O_{2}\left(N^{x}\right)\right)$ char $N^{x}$ and so $Z\left(O_{2}\left(N^{x}\right) \triangleleft G_{x}\right.$. Now $Z\left(O_{2}\left(N^{x}\right)\right)$ contains some involution and by Lemma $2.2, G_{x}$ acts transitively by conjugation on the involutions of $N^{x}$. Thus all involutions of $N^{x}$ belong to $\mathrm{Z}\left(\mathrm{O}_{2}\left(\mathrm{~N}^{x}\right)\right)$ and so commute.

LEMMA 2.12. Let $K(x, y)$ be a strongly closed subgroup of $G_{x y}$ and suppose $|K(x, y)|$ is odd. Then either

(i) $K(x, y)$ fixes exactly two points, or

(ii) $O_{2}\left(N^{x}\right) \neq 1$ and $\left[\Omega_{1}\left(Z\left(O_{2}\left(N^{x}\right)\right)\right), K(x, y)\right]=1$.

PRoOF. With $\Gamma_{K}(x, y)=F_{K(x, y)}$, by Lemma 1.8 , the family of sets $B=\left\{\Gamma_{K}(x, y)\right\}$ forms a block design on $X$ preserved by $G$. Suppose $K(x, y)$ fixes more than two points, so $\left|\Gamma_{K}(x, y)\right|>2$. Set $B=\Gamma_{K}(x, y)$.

By Lemma 1.11, $W(x, y)=\Pi_{(u, v) \in B \times B-\delta(B)} K(u, v)$ is a weakly closed supgroup of $G_{x y}$ in $G,|W(x, y)|$ is odd, as each $|K(u, v)|$ is odd, and $F_{W(x, y)}=$ $B$. By Lemma $2.7,|B|$ is odd.

Let $T^{x}$ be the subgroup of $N^{x}$ which fixes all blocks of $B_{x}$. Then $T^{x} \triangleleft$ $G_{x}$ and by Lemma $2.10, T^{x}$ contains all involutions of $N^{x}$. If $B^{\prime} \in B_{x}$, then $G_{B^{\prime}} \mid B^{\prime}$ is doubly-transitive and $T^{x} \mid B^{\prime} \triangleleft\left(G_{B^{\prime}}^{*} \mid B^{\prime}\right)_{x}$. Since $T^{x} \subseteq N^{x}, T_{y}^{x}$ is nilpotent if $y \in X-x$. By induction all involutions of $T^{x} \mid B^{\prime}$ commute. Let $k_{1}$ and $k_{2}$ be two involutions of $N^{x}$. Then $k_{1}, k_{2} \in T^{x}$ and $\left[k_{1}, k_{2}\right] \mid B^{\prime}=1$. Since $B^{\prime}$ is any block of $B_{x},\left[k_{1}, k_{2}\right]=1$. Thus, by Lemma $2.11, O_{2}\left(N^{x}\right) \neq 1$ and $\Omega_{1}\left(Z\left(O_{2}\left(N^{x}\right)\right)\right)$ fixes all blocks of $B_{x}$.

Since $\Omega_{1}\left(Z\left(O_{2}\left(N^{x}\right)\right)\right)$ fixes $B,\left[\Omega_{1}\left(Z\left(O_{2}\left(N^{x}\right)\right)\right), G_{B}\right] \subseteq G_{B}$. Since $\Omega_{1}\left(Z\left(O_{2}\left(N^{x}\right)\right)\right) \triangleleft G_{x}$ and $G_{B} \subseteq G_{x},\left[\Omega_{1}\left(Z\left(O_{2}\left(N^{x}\right)\right)\right), G_{B}\right] \subseteq \Omega_{1}\left(Z\left(O_{2}\left(N^{x}\right)\right)\right)$. Since all involutions of $N^{x}$ fix only $x$ and $|B| \geqslant 3, \Omega_{1}\left(Z\left(O_{2}\left(N^{x}\right)\right)\right) \cap G_{B}=1$. Since $K(x, y) \subseteq G_{B}, \quad\left[\Omega_{1}\left(Z\left(O_{2}\left(N^{x}\right)\right)\right), K(x, y)\right]=1$.

Immediately from Lemma 2.12 we have

Lemma 2.13. Let $P(x, y)$ be the Sylow p-subgroup of $N_{y}^{x}$. Then either

(i) $P(x, y)$ fixes exactly two points, or

(ii) $O_{2}\left(N^{x}\right) \neq 1$ and $\left[\Omega_{1}\left(Z\left(O_{2}\left(N^{x}\right)\right)\right), P(x, y)\right]=1$.

In Lemmas 2.13 to 2.17 we assume $O_{2}\left(N^{x}\right)=1$ and obtain a contradiction. Already by Lemma 2.13 we know that each Sylow subgroup of $N_{y}^{x}$ fixes exactly two points. 
LEMMA 2.14. $N_{y}^{x}$ is a p-group.

Proof. Let $P(x, y)$ be the Sylow $p$-subgroup of $N_{y}^{x}$. By hypothesis $P(x, y) \triangleleft N_{y}^{x}$. Since $P(x, y)$ fixes exactly $x$ and $y, N_{N^{x}}(P(x, y))=N_{y}^{x}$. Thus, $N_{y}^{x}$ is a nilpotent group which is the normalizer of each of its Sylow subgroups. By a result of Wielandt [17], if $\left|N_{y}^{x}\right|$ has two distinct prime factors, $N_{y}^{x}$ has a normal complement $K^{x}$ in $N^{x}$. Then $K^{x}$ char $N^{x}$ and so $K^{x} \triangleleft G_{x}$. Also, $K^{x}$ is semiregular on $X-x$. By Corollary $\mathrm{Cl}, \mathrm{O}_{2}\left(K^{x}\right) \neq 1$, and so $\mathrm{O}_{2}\left(N^{x}\right) \neq 1$, contrary to hypothesis.

LEMMA 2.15. $p \mid s$.

Proof. Since $G_{x y}$ fixes the set $\Lambda(x, y) \subseteq X-\{x, y\}, N_{y}^{x}$ fixes this set. Since $N_{y}^{x}$ is a $p$-group and fixes exactly the points $x$ and $y, p|| \Lambda(x, y) \mid$ and so $p \mid s$.

LEMMA 2.16. $O\left(N^{x}\right)=1$.

Proof. Suppose not and take $A^{x}$ a minimal characteristic subgroup of $O\left(N^{x}\right)$. By the Feit-Thompson theorem, $A^{x}$ is elementary abelian of order some power of $r$, where $r$ is an odd prime.

Since $A^{x} \triangleleft G_{x}, r \mid n$. Since $p \mid s$, by Lemma $2.8, p \mid n-1$. Thus, $p \neq r$.

Since $N^{x}=N_{y}^{x} C_{N^{x}}(j)$ and $N_{y}^{x}$ is a $p$-group, $j$ has $p^{r}$ conjugates under $N^{x}$. Since $A^{x} \triangleleft N^{x}$ and $r \neq p, A^{x}$ centralizes all conjugates of $j$ under $N^{x}$. Thus, $M^{x}=C_{N^{x}}\left(A^{x}\right)$ is of even order. As $A^{x}$ char $N^{x}, M^{x}$ char $N^{x}$ and $M^{x} \triangleleft G_{x}$.

We claim that $M^{x}$ is semiregular on $X-x$.

If not, $M_{y}^{x} \neq 1$, for $y \in X-x$. Then, since $A^{x}$ centralizes $M_{y}^{x}, \Delta_{A}(x, y)$ $\subseteq \Gamma_{M}(x, y) \subset X$. Thus, $\left|\Gamma_{M}(x, y)\right| \geqslant 1+r$. Also, $M_{y}^{x}$ is a strongly closed subgroup of $G_{x y}$ in $G_{x}$. By Lemma 2.12, a contradiction results for $O_{2}\left(N^{x}\right)=1$.

Since $M^{x} \triangleleft G_{x},\left|M^{x}\right|$ is even, and $M^{x}$ is semiregular, by Corollary $\mathrm{C} 1$, $O_{2}\left(M^{x}\right) \neq 1$. Again a contradiction results. Thus, $O\left(N^{x}\right)=1$.

LEMma 2.17. Take $K^{x}$ a minimal characteristic subgroup of $N^{x}$. Then $K^{x}$ is a nonabelian simple group.

Proof. Since $O\left(N^{x}\right)=1$ and $O_{2}\left(N^{x}\right)=1, K^{x}$ is the direct product of isomorphic, nonabelian simple groups, and $K^{x}$ is of even order. Since $G_{x}$ acts transitively by conjugation on the involutions of $N^{x}$ it acts transitively on those of $K^{\boldsymbol{x}}$. Thus, $K^{\boldsymbol{x}}$ has only one direct factor.

We now obtain a final contradiction to $O_{2}\left(N^{x}\right) \neq 1$. Clearly, $K_{y}^{x}$ is a $p$-group. By Theorem $\mathrm{C}(\mathrm{i}), K^{x}=C_{K^{x}}(j) K_{y}^{x}$. Thus, $j$ has $p^{\alpha}$ conjugates under $K^{x}$. By a theorem of Burnside [5, p. 322], $j$ is central in some homomorphic image of $K^{x}$, in contradiction to the simplicity of $K^{x}$. It follows: 
THEOREM C(ii). If $N_{y}^{x}$ is nilpotent, $O_{2}\left(N^{x}\right) \neq 1$.

3. $N^{x}$ a T.I. set. In this section we assume $G$ is a doubly-transitive group on a set $X, x \in X$, and $N^{x}$ is a normal subgroup of $G_{x}$. In addition, we assume

(i) $N^{x}$ is a T.I. set, and

(ii) $N^{x}$ is not semiregular on $X-x$.

As usual, $N^{y}$ is defined for other $y \in X$, so that $f N^{y} f^{-1}=N^{f(y)}$. By (i), $N^{x} \cap N^{y}=1$ if $x \neq y$. In addition, if $y \in X-x, N_{y}^{x} \neq 1$, by (ii).

In this section we derive most of the fundamental facts which are used in the proof of Theorem A.

Lemma 3.1. If $A \subseteq N^{x}, A \neq 1, N_{G}(A) \subseteq G_{x}$.

Proof. Take $f \in N_{G}(A)$. Then, $A \subseteq N^{x} \cap N^{f(x)}$. Thus, if $f(x) \neq x$, $N^{x} \cap N^{f(x)} \neq 1$, a contradiction. Thus, $f(x)=x$ and $N_{G}(A) \subseteq G_{x}$.

Lemma 3.2. $N_{y}^{x} \triangleleft G_{x y}, N_{x}^{y} \triangleleft G_{x y}$, and $\left[N_{y}^{x}, N_{x}^{y}\right]=1$.

PROOF. $N_{y}^{x}=N^{x} \cap G_{y x} \triangleleft G_{x} \cap G_{y x}=G_{x y}$. Likewise, $N_{x}^{y} \triangleleft G_{x y}$. Since $N_{y}^{x} \cap N_{x}^{y} \subseteq N^{x} \cap N^{y}=1,\left[N_{y}^{x}, N_{x}^{y}\right]=1$.

The following is the foundation on which most of the subsequent analysis is based.

LEMMA 3.3. $N_{x}^{y}$ normalizes $N^{x}$. If $f \in N_{x}^{y}, f \neq 1, C_{N^{x}}(f)=N_{y}^{x}$.

Proof. Since $N_{x}^{y} \subseteq G_{x}, N_{x}^{y}$ normalizes $N_{y}^{x}$. By Lemma 3.2, $\left[N_{x}^{y}, N_{y}^{x}\right]=1$. Thus, $N_{y}^{x} \subseteq C_{N^{x}}(f)$. On the other hand, as $f \neq 1$, by Lemma 3.1, $C_{N^{x}}(f) \subseteq$ $N^{x} \cap G_{y}=N_{y}^{x}$.

LEMmA 3.4. Suppose that $K(x, y) \subseteq N_{y}^{x}$ and $K(x, y)$ is a strongly closed subgroup of $G_{x y}$ in $G_{x}$. Suppose also that $K(x, y) K(y, x)$ is a weakly closed subgroup of $N_{y}^{x} K(y, x)$ in $N^{x} K(y, x)$. Then:

(i) $N_{N^{x}}(K(x, y))=N_{N^{x}}(K(x, y) K(y, x))$.

(ii) $K(y, x)$ normalizes $N_{N}(K(x, y))$ and acts trivially on

$$
N_{N^{x}}(K(x, y)) / K(x, y) .
$$

Proof. This is immediate from Lemmas 1.13 and 1.15, since in this case, $K(y, x) \cap N^{x} \subseteq N^{y} \cap N^{x}=1 \subseteq K(x, y)$.

In particular, it follows that

LEMmA 3.5. $N_{N} x\left(N_{y}^{x}\right)=N_{N} x\left(N_{y}^{x} N_{x}^{y}\right)$ and $N_{x}^{y}$ acts trivially on $N_{N^{x}}\left(N_{y}^{x}\right) / N_{y}^{x}$.

LEMMA 3.6. Suppose $N_{y}^{x}$ is an abelian group and $P(x, y)$ the Sylow p-subgroup of $N_{y}^{x}$ and $P_{i}(x, y)=\Omega_{i}(P(x, y))$. Then $N_{N^{x}}\left(P_{i}(x, y)\right)=N_{N^{x}}\left(N_{y}^{x}\right)$. 
Proof. Since $P_{i}(x, y) \operatorname{char} N_{y}^{x}, N_{N^{x}}\left(N_{y}^{x}\right) \subseteq N_{N^{x}}\left(P_{i}(x, y)\right)$. To prove the opposite inclusion we first show, using Lemma 3.4, that $N_{N} x\left(P_{i}(x, y) P_{i}(y, x)\right)=$ $N_{N^{x}}\left(P_{i}(x, y)\right)$.

We first show that $P_{i}(x, y)$ is a strongly closed subgroup of $G_{x y}$ in $G_{x}$. Suppose $g \in G_{x}$. Then $g P_{i}(x, y) g^{-1} \subseteq N^{x}$, as $P_{i}(x, y) \subseteq N^{x}$ and $N^{x} \triangleleft G_{x}$. Thus, $G_{x y} \cap\left(g P_{i}(x, y) g^{-1}\right) \subseteq G_{x y} \cap N^{x}=N_{y}^{x}$. But $g P_{i}(x, y) g^{-1}$ is a $p$-group of exponent $p^{i}$, and so $G_{x y} \cap\left(g P_{i}(x, y) g^{-1}\right) \subseteq P_{i}(x, y)$. Thus, $P_{i}(x, y)$ is a strongly closed subgroup of $G_{x y}$ in $G_{x}$,

Now $\left[N_{y}^{x}, N_{x}^{y}\right]=1$, by Lemma 3.2 , and so, as $P_{i}(y, x) \subseteq N_{x}^{y}$, $P_{i}(x, y) P_{i}(y, x)$ is $\Omega_{i}$ of a Sylow $p$-subgroup of $N_{y}^{x} P_{i}(y, x) \cong N_{y}^{x} \times P_{i}(y, x)$. Thus, $P_{i}(x, y) P_{i}(y, x)$ is a weakly closed subgroup of $N_{y}^{x} P_{i}(y, x)$ in $N^{x} P_{i}(y, x)$.

By Lemma 3.4, $N_{N^{x}}\left(P_{i}(x, y) P_{i}(y, x)\right)=N_{N^{x}}\left(P_{i}(x, y)\right)$. Thus $N_{N^{x}}\left(P_{i}(x, y)\right)$ normalizes $C_{N^{x}}\left(P_{i}(x, y) P_{i}(y, x)\right)$. By Lemma 3.3, $C_{N^{x}}\left(P_{i}(y, x)\right)=N_{y}^{x}$. Since $N_{y}^{x}$ is abelian, $C_{N^{x}}\left(P_{i}(x, y) P_{i}(y, x)\right)=N_{y}^{x}$. Thus $N_{N^{x}}\left(P_{i}(x, y)\right)$ normalizes $N_{y}^{x}$ and so $N_{N^{x}}\left(P_{i}(x, y)\right) \subseteq N_{N} x\left(N_{y}^{x}\right)$, and Lemma 3.6 follows.

In what follows we essentially reduce the structure of $N^{x}$ to one of two cases. In the first of these cases $N^{x}$ has an abelian characteristic subgroup $A^{x}$ and $A^{x} \cap N_{y}^{x} \neq 1$. Thus, $G_{x}$ has an abelian normal subgroup which is not semiregular on $X-x$. By Theorem A of [11], it follows that $\operatorname{PSL}(n, q) \subseteq G \subseteq$ $P \Gamma L(n, q)$, and we obtain our Theorem A. In the second case, replacing $N^{x}$ by a characteristic subgroup $K^{x}$, we may assume that $K_{y}^{x}$ is cyclic of prime order, $K_{y}^{x}$ is a Sylow $p$-subgroup of $K^{x}$ and has a normal complement in $K^{x}$. We shall show next that this latter case leads to a contradiction.

LEMMA 3.7. $G$ does not have a regular normal subgroup. $G_{x}$ considered as a permutation group on $X-x$ does not have a regular normal subgroup.

Proof. Suppose $G$ has a regular normal subgroup $A$. Then $A \cdot N^{x} \triangleleft G$, as $N^{x} \triangleleft G_{x}$. Now $\left(A \cdot N^{x}\right)_{x}=N^{x}$, since $A$ is regular. Thus, $\left(A \cdot N^{x}\right)_{x y}=N_{y}^{x}$. Since $A$ is transitive, $A \cdot N^{x}=A N^{y}$. Thus, $\left(A N^{y}\right)_{y}=N^{y}$ and $\left(A N^{y}\right)_{x y}=N_{x}^{y}$. Thus, $N_{y}^{x}=N_{x}^{y}$. Since $N_{y}^{x} \neq 1$ and $N^{x} \cap N^{y}=1$, this is impossible.

Suppose next that $G_{x}$ has a regular normal subgroup. Since $G$ does not have a regular normal subgroup, it follows from results of Hering, Kantor, Seitz [10] and Shult [13], that the minimal normal subgroup of $G$ is $\operatorname{PSL}(2, q)$, $\operatorname{Sz}(q), \operatorname{PSU}(3, q)$, or a group of Ree type. In all of these cases, the two point stabilizer of $G$ is some subgroup of the holomorph of a field having $r$ elements $\left(r=q^{2}\right.$, if $\operatorname{PSU}(3, q) \triangleleft G$, otherwise $\left.r=q\right)$. Moreover, the two point stabilizer of $G$ has a cyclic normal subgroup $T$, where $|T| \geqslant(r-1) / 3$. Since $N_{y}^{x} N_{x}^{y} \triangleleft G_{x y}$ and $N_{y}^{x} \cap N_{x}^{y}=1$, it follows that $N_{y}^{x}$ is cyclic, and as we wish to show $N^{x} \cap$ $N^{y} \neq 1$, we may assume $N_{y}^{x}$ is cyclic of prime order.

If $N_{y}^{x} \cap T=1$, since $N_{y}^{x} \triangleleft G_{x y}, N_{y}^{x}$ centralizes $T$. Since $N_{y}^{x}$ induces a 
field automorphism of $T,(r-1) / 3 \leqslant r^{1 / 2}-1$. Thus, $r^{1 / 2}+1 \leqslant 3$, or $r \leqslant 4$. Since $G$ has no regular normal subgroup, it follows that $\operatorname{PSL}(2,4) \subseteq G \subseteq$ $P \Gamma L(2,4)$, or $G=P \Gamma L(2,8)$, the smallest Ree group. In these cases, clearly $N_{y}^{x}=N_{x}^{y}$.

Thus $N_{y}^{x} \subseteq T$. Likewise, $N_{x}^{y} \subseteq T$. So $N_{y}^{x}=N_{x}^{y}$, contrary to $N^{x} \cap N^{y}=1$.

In the remainder of this section we assume that $N_{y}^{x}$ is cyclic of prime order $p$, that $N_{y}^{x}$ is a Sylow $p$-subgroup of $N^{x}$, and that $N_{y}^{x}$ has a normal complement $M^{x}$ in $N^{x}$. We assume $N^{x} \cap N^{y}=1$ and derive a contradiction.

Clearly, $M^{x} \triangleleft G_{x}$ and $M^{x}$ is semiregular on $X-x$.

LEMmA 3.8. $N^{x}$ is a Frobenius group with kernel $M^{x}$ and complement $N_{y}^{x}$.

Proof. It suffices to show $C_{M^{x}}\left(N_{y}^{x}\right)=1$. Set $T=C_{M^{x}}\left(N_{y}^{x}\right)$. Since $M^{x}$ $\triangleleft G_{x}, N_{x}^{y}$ normalizes $M^{x}$. Since $N_{x}^{y}$ normalizes $N_{y}^{x}, N_{x}^{y}$ normalizes $T$. Thus, $\left[T, N_{x}^{y}\right] \subseteq T$.

On the other hand, by Lemma 3.5, $\left[N_{x}^{y}, N_{N^{x}}\left(N_{y}^{x}\right)\right] \subseteq N_{y}^{x}$. Thus, $\left[N_{x}^{y}, T\right]$ $\subseteq N_{y}^{x}$. Since $T \cap N_{y}^{x} \subseteq M^{x} \cap N_{y}^{x}=1,\left[N_{x}^{y}, T\right]=1$. By Lemma 3.3, $T \subseteq N_{y}^{x}$, and so as $T \cap N_{y}^{x}=1, T=1$.

Now by Lemma 1.7 the fixed point sets $\left\{\Gamma_{N}(x, y)\right\}$ form a block design $B$ on $X$ preserved by $G$. We suppose $|X|=1+n$, if $B \in B,|B|=1+l$, and $\left|M^{x}\right|=m$. Thus, since $N_{y}^{x}$ fixes exactly one point of each orbit of $M^{x}, n=m l$. For the same reason it follows that $M^{x}$ is sharply transitive on the blocks of $B_{x}$. By Lemma 3.7, we may assume $l \geqslant 2$. Thus, $B$ is a proper block design.

Lemma 3.9. $C_{G_{x}}\left(M^{x}\right)$ is semiregular on $X-x$.

Proof. Suppose not. By Lemma 1.17, there is a proper $M$-admissible block design $C$. Suppose if $C \in C,|C|=1+k$. Since $M^{x}$ fixes all blocks on $C_{x}, m \leqslant k$. Thus, $n \leqslant l k$.

Since the block designs $B$ and $C$ are proper, by Lemma 1.1(iii), $1+k \leqslant$ $n / k$ and $1+l \leqslant n / l$. Taking $d$ as the larger of the numbers $k$ and $l$, it follows that $n / d \leqslant d$ and $1+d \leqslant n / d$, a contradiction, and Lemma 3.9 follows.

LEMmA 3.10. Let $B$ be $a$ block of $B$ and $V$ a subgroup of $G_{B}$. Then

(i) $F_{V}$ is a subspace over $B, C_{G_{x}}(V)$ is transitive on the blocks of $B_{x}$ contained in $F_{V}$ for each $x \in B$, and $C_{G}(V)$ is transitive on the blocks of $B$ contained in $F_{V}$.

(ii) If $B \subset F_{V}, C_{G}(V)$ is transitive on $F_{V}$.

Proof. Take $x \in B$. Since $V$ fixes $x, V$ normalizes $M^{x}$. We consider the action of $M^{x} V$ on the blocks of $B_{x}$. Since $M^{x}$ is sharply transitive on the blocks of $B_{x}, V$ is the subgroup of $M^{x} V$ fixing the set $B \in B_{x}$. Now the representation of $M^{x} V$ on the blocks of $B_{x}$ is faithful. Indeed, let $T$ be the 
subgroup of $M^{x} V$ fixing all blocks of $B_{x}$. Then $T \subseteq V$ as $T$ fixes $B$, and $T \triangleleft$ $M^{x} V$. Thus $N(T)$ is transitive on the blocks of $B_{x}$ and $T$ fixes all points of $B$, so $T$ fixes all points of $X$, and so $T=1$.

Therefore, $M^{x} V$ acts faithfully on the blocks of $B_{x}$ and has a regular normal subgroup $M^{x}$. Thus, $C_{M^{x}}(V)$ is transitive on the blocks of $B_{x}$ fixed by $V$. Thus, if $y$ is any point of $F_{V}, B(x, y) \subseteq F_{V}$, as $V$ fixes $B(x, y)$.

Therefore, any point $y$ of $F_{V}$ is contained in some block $C$ contained in $F_{V}$. By the same reasoning if $z \in F_{V}-y, B(y, z) \subseteq F_{V}$. Therefore, $F_{V}$ is a subspace over $B$ and $C_{M} x(V)$ is transitive on the blocks of $B_{x}$ contained in $F_{V}$, for any $x \in F_{V}$. Thus, $C_{G}(V)$ is transitive on the blocks of $B$ in $F_{V}$.

Suppose now $B \subset F_{V}$. Let $t$ be the number of blocks of $B_{x}$ contained in $F_{V}$, for $x \in F_{V}$. Then $\left|C_{M^{x}}(V)\right|=t>1$, as $B \subset F_{V}$. Moreover, $C_{M^{x}}(V)$ is semiregular on $F_{V}-x$. Since this is true for each $x \in F_{V}$, it follows (see e.g. [11, Lemma 4.9]) that $C_{G}(V)$ is transitive on $F_{V}$.

LEMmA 3.11. Let $B$ be a block of $B, x \in B$, and $V$ a subgroup of $G_{B}$. Set $H=G_{B}^{*}$. If $C_{M^{x}}(V) \neq 1$, then $C_{H}(V)$ is transitive on $B$.

Proof. Since $V$ fixes all points of $B$ and $C_{M} x(V) \neq 1, F_{V} \supset B$. Let $L=C_{G}(V) \mid F_{V}$. By Lemma 3.10, $L$ is transitive on $F_{V}$ and $L_{x}$ on the blocks of $B_{x}$ contained in $F_{V}$ for each $x \in F_{V}$. We show that $L_{B}^{*}$ is transitive on $B$.

Let $\left|F_{V}\right|=1+m$ and $|B|=1+l$. Then $\left|L: L_{x}\right|=1+m$, and by Lemma 1.1(i) and (ii), $\left|L_{x}:\left(L_{x}\right)_{B}^{*}\right|=m / l$ and $\left|L: L_{B}^{*}\right|=(1+m) m /(1+l) l$. Thus, $(1+l)\left|L: L_{B}^{*}\right|=\left|L: L_{x}\right|\left|L_{x}:\left(L_{x}\right)_{B}^{*}\right|=\left|L:\left(L_{x}\right)_{B}^{*}\right|$. So $1+l=\left|L_{B}^{*}:\left(L_{B}^{*}\right)_{x}\right|$.

Now we fix a block $B$ containing $x$ and $y$. We let $W$ be the weak closure of $N_{y}^{x}$ in $G_{x y}$. By Lemma 1.11,W= $\Pi_{(u, v) \in B \times B-\delta(B)} N_{v}^{u}$. Since $N_{v}^{u} \triangleleft W$, if $(u, v) \in B \times B-\delta(B)$ and $N_{v}^{u}$ is cyclic of order $p, W$ is an elementary abelian $p$-group. Since $F_{w}=B, N_{v}^{u}=N_{v^{\prime}}^{u}$, if $(u, v),\left(u, v^{\prime}\right) \in B \times B-\delta(B)$. Therefore, for each $u \in B$, there is a unique $N_{v}^{u}$ contained in $W$. If $(u, v) \in B \times B-\delta(B)$, we take $U_{u}=N_{v}^{u}$. The collection $U_{u}, u \in B$, we denote by $U$. Since $N^{x} \cap$ $N^{y}=1$ if $x \neq y,|U|=|B|=1+l$. Since $l \geqslant 2, W$ is noncyclic and spanned by $U$.

Set $H_{1}=G_{B}^{*}$. Clearly, $W \triangleleft H$. Moreover, the action of $H$ on $B$ is equivalent to its action on $U$ induced by conjugation of $W$. We let $\bar{H}=H / C_{H}(W)$.

By $V$ we mean the collection of maximal subgroups $V$ of $W$ such that $C_{M} x(V) \neq 1$.

LEMmA 3.12. If $U \in U$ and $V \in V$, then $U \cap V=1$.

Proof. It is sufficient to show that $C_{M^{x}}(U)=1$. Indeed, if $U=N_{y}^{x}$, $C_{M} x(U)=1$ by Lemma 3.8. If $U=N_{x}^{z}$ for some $z \neq x$, then since $C_{G}\left(N_{x}^{z}\right) \subseteq$ $G_{z}$ by Lemma 3.3 and $M^{x}$ is semiregular on $X-x$, again $C_{M} x(U)=1$. 
Lemma 3.13. $\bigcap_{V \in V} V=1$.

Proof. Set $T=\bigcap_{V \in V} V$. Since $N_{y}^{x}$ acts without fixed points on $M^{x}$, by Thompson's theorem, $M^{x}$ is a nilpotent $p^{\prime}$-group. Take $R_{0} \subseteq R_{1} \subseteq \ldots \subseteq$ $R_{k}=M^{x}$, a chief series for $M^{x} W$ passing through $M^{x}$. Then, $R_{i+1} / R_{i}$ is abelian and $W$ induces a cyclic group on $R_{i+1} / R_{i}$, and the kernel of the action is some $V \in V$, as $M^{x}$ is a $p^{\prime}$-group. Since $T \subseteq W \subseteq G_{x y}$, by Lemma 3.9, $T=1$.

LEMMA 3.14. $C_{\bar{H}}(V)$ is transitive on $U$ for each $V \in V$.

This is immediate from Lemma 3.11.

We let $E$ be the field with $p$ elements, $E^{\#}$ its multiplicative subgroup.

LEMMA 3.15. Let $V$ be a maximal subgroup of $W$ and $K$ a subgroup of Aut $(W)$ which centralizes $V$. Let $\Omega$ be an orbit of $K$ on $W-V$.

Then, there is a subgroup $P_{V}$ of $V$, a point $x \in V$ and $y \in W-V$, and $a$ subgroup $D$ of $E^{\#}$, such that $\Omega=\left\{x+\lambda y+P_{V} \mid \lambda \in D\right\}$. Moreover, if $\Omega$ spans $W,\left|W: P_{V}\right| \leqslant p^{2}$.

Proof. Suppose $|W|=p^{r}$, and $L$ is the subgroup of $\operatorname{Aut}(W)$ which centralizes $V$. Then $L$ has a normal subgroup of $Q$ of order $p^{r-1}$ consisting of linear transformations of the form $T_{u}(z)=z+f(z) u$, where $f$ is a fixed nonzero linear functional of $W$ which annihilates $V$ and $u$ is any element of $V$. Then $T_{u_{1}}+T_{u_{2}}=T_{u_{1}+u_{2}}$ and $L / Q \cong E^{\#}$.

Let $R=K \cap Q$. Then there is a subgroup $P_{V}$ of $V$ such that $R=$ $\left\{T_{u} \mid u \in P_{V}\right\}$. Also, $K=R \cdot D_{0}$, where $D_{0}$ is cyclic of order dividing $(p-1)$. Then there is a point $y \in W-V$ and a subgroup $D \subseteq E^{\#}$, such that $D_{0}$ consists of the transformations $S_{\lambda}, \lambda \in D$, and $S_{\lambda}(y)=\lambda y$, and $S_{\lambda}(z)=z$ for $z \in V$.

The orbit of $y$ under $K$ is then $\left\{\lambda y+P_{V} \mid \lambda \in D\right\}$. If now $t \in \Omega, t=$ $x+\mu y$, for some $x \in V$ and $\mu \in E^{\#}$. Since the transformations of $K$ fix $x$, replacing $y$ by $\mu y, \Omega=\left\{x+\lambda y+P_{V} \mid \lambda \in D\right\}$.

Since $P_{V}$ is a subgroup of $W$, if $\Omega$ spans $W,\left|W: P_{V}\right| \leqslant p^{2}$.

LEMMA 3.16. Suppose $V_{1}, V_{2} \in V$ and set $K_{1}=C_{\bar{H}}\left(V_{1}\right)$ and $K_{2}=C_{\bar{H}}\left(V_{2}\right)$. With $P_{V_{1}}$ and $P_{V_{2}}$ as in Lemma 3.15, we have $P_{V_{1}}=P_{V_{2}}$.

Proof. Let $u \in U \in U$. We consider the orbits $\Omega_{1}$ and $\Omega_{2}$ of $K_{1}$ and $K_{2}$ on $u$. By Lemma 3.14, $K_{1}$ and $K_{2}$ are transitive on $U$ and so $\left\{\mu \Omega_{1} \mid \mu \in E^{\#}\right\}$ $=\left\{\mu \Omega_{2} \mid \mu \in E^{\#}\right\}$. With $x_{1}, y_{1}, x_{2}, y_{2}, D_{1}, D_{2}$, as in Lemma 3.15 , it follows that

$\left\{\mu x_{1}+\lambda \mu y_{1}+P_{V_{1}} \mid \mu \in E^{\#}, \lambda \in D_{1}\right\}=\left\{\mu x_{2}+\lambda \mu y_{2}+P_{V_{2}} \mid \mu \in E^{\#}, \lambda \in D_{2}\right\}$. 
We consider the image of each of these sets in $W / P_{V_{1}}$. If $b$ and $B$ are elements or sets of $W$, we denote their images in $W / P_{V_{1}}$ by $\bar{b}$ and $\bar{B}$. The image of the first set is $\left\{\mu \bar{x}_{1}+\lambda \mu \bar{y}_{1} \mid \mu \in E^{\#}, \lambda \in D_{1}\right\}$. If $x_{1} \in P_{V_{1}}$, this set has $p-1$ elements. Otherwise, as $y_{1} \notin\left\langle x, P_{V_{1}}\right\rangle \subseteq V_{1}$, the set has $(p-1)\left|D_{1}\right|$ elements. The image of the second set is $\left\{\mu \bar{x}_{2}+\lambda \mu \bar{y}_{2}+\bar{P}_{V_{2}} \mid \mu \in E^{\#}, \lambda \in D_{2}\right\}$. If $t$ belongs to this set, so does $t+\bar{P}_{V_{2}}$. Thus, if $P_{V_{2}} \stackrel{\Phi}{P_{V_{1}}}$, the number of elements of the second set is divisible by $p$, a contradiction as both sets have the same number of elements. Thus, $P_{V_{2}} \subseteq P_{V_{1}}$. Likewise, $P_{V_{1}} \subseteq P_{V_{2}}$ and Lemma 3.16 follows.

LEMMA 3.17. $|W|=p^{2}$ and $P_{V}=1$ if $V \in V$.

Proof. If for some $V_{0} \in V, P_{V_{0}} \neq 1$, then for all $V \in V, P_{V}=P_{V_{0}}$, by Lemma 3.16, and since $P_{V} \subseteq V, P_{V_{0}} \subseteq \bigcap_{V \in V} V$. By Lemma 3.13 this is impossible. Thus, $P_{V}=1$ if $V \in V$. Since $U$ spans $W$, by Lemma $3.15,|W|=p^{2}$.

LEMMA 3.18. (i) $l=2$.

(ii) $|V|=2$.

(iii) $V_{1}, V_{2} \in V$ are conjugate in $G$.

(iv) If $V \in V, V \triangleleft G_{x y}$.

Proof. By Lemma 3.17, $|W|=p^{2}$ and so $\bar{H} \subseteq G L(2, p)$. Moreover, $\bar{H}$ is doubly-transitive on the family of one-dimensional subspaces $U$ of $W$.

We claim that $p$ does not divide $|\bar{H}|$. Indeed, if $p|| \bar{H} \mid$, since $|U|=1+l \geqslant$ 3 , either $|U|=p$ or $1+p$. Thus, $|V| \leqslant 1$, a contradiction by Lemma 3.13.

Since $|U| \geqslant 3$, the subgroup of $\bar{H}$ fixing all elements of $U$ fixes all onedimensional subspaces and is contained in $Z(\bar{H})$. Thus, $L=\bar{H} / Z(\bar{H})$ is doublytransitive on $U$. Since $p \backslash|L|$, the stabilizer in $L$ of $U \in U$ is cyclic. Moreover, if $V \in V$, since $p \gamma|\bar{H}|$ and $C_{\bar{H}}(V)$ is transitive on $U, L$ has a cyclic regular subgroup, and $(1+l) \mid(p-1)$. Thus, $L$ is a Frobenius group with cyclic kernel and complement. By the local structure of $\operatorname{PGL}(2, p)$ and since $1+l \geqslant 3$, $\bar{H} / Z(\bar{H}) \cong S_{3}$ and $l=2$.

Now for each $V \in V, C_{\bar{H}}(V)$ induces the 3-element of $L$ by restriction to $U$ and the three element of $L$ fixes precisely two one-dimensional subspaces. Thus, $|V| \leqslant 2$. By Lemma $3.13,|V|=2$. Also any two element which inverts the 3-element of $S_{3}$ interchanges $V_{1}$ and $V_{2}$ and so $V_{1}$ and $V_{2}$ are conjugates.

Since $G_{x y} \subseteq H$ and $G_{x y}$ fixes $U_{x}$ and $U_{y}, G_{x y}$ fixes all points of $U$ and so normalizes $V_{1}$ and $V_{2}$, completing the proof of the lemma.

We now obtain a contradiction to $N^{x} \cap N^{y}=1$ if $x \neq y$. Take $M_{1}=$ $C_{M^{x}}\left(V_{1}\right)$ and $M_{2}=C_{M^{x}}\left(V_{2}\right), V_{1}, V_{2}$ as in Lemma 2.18. Since $V_{1}$ and $V_{2}$ are the only cyclic subgroups of $W$ which centralize nonidentity elements of $M^{x}, M^{x}=M_{1} M_{2}$ and $M_{1} \cap M_{2}=1$. Since $V_{1}$ and $V_{2}$ are conjugates and 
$C_{G}\left(V_{1}\right)$ is transitive on $F_{V_{1}},\left|M_{1}\right|=\left|M_{2}\right|$. Let $m=\left|M_{1}\right|$. Since $l=2,|X|=1+$ $2 m^{2}$ and $\left|F_{V}\right|=1+2 m$. Since $C_{G}\left(V_{1}\right)$ is transitive on $F_{V_{1}}, M_{1} \subseteq C_{G}\left(V_{1}\right)$ and $V_{1} \triangleleft G_{x y},(1+2 m) m\left|G_{x y}\right||| N_{G}\left(V_{1}\right) \mid$. Thus

$$
(1+2 m) m\left|G_{x y}\right|\left|\left(1+2 m^{2}\right)\left(2 m^{2}\right)\right| G_{x y} \mid,
$$

and so $(1+2 m) \mid\left(1+2 m^{2}\right)(2 m)$. It follows that $(1+2 m) \mid(m-1)$, a contradiction.

From this contradiction and the remarks preceding Lemma 3.7, the first two statements of the next lemma follows:

LEMmA 3.19. Theorem A follows if (with $y \in X-x$ ):

(i) $N^{x}$ has a characteristic abelian subgroup $K^{x}$ with $K_{y}^{x} \neq 1$, or

(ii) $N^{x}$ has a characteristic subgroup $K^{x}$ with $K_{y}^{x}$ cyclic of prime order, $K_{y}^{x}$ a Sylow subgroup of $K^{x}$ and having a normal complement in $K^{x}$, or

(iii) $N^{x}$ is of even order, $N_{y}^{x}$ abelian of odd order.

Proof. We need only prove (iii). By Theorem $\mathrm{C}, O_{2}\left(N^{x}\right) \neq 1$. Suppose $P(x, y)$ is a Sylow $p$-subgroup of $N_{y}^{x}$. Suppose first that $P(x, y)$ fixes more than two points. By Lemma $2.13,\left[\Omega_{1}\left(Z\left(O_{2}\left(N^{x}\right)\right), P(x, y)\right]=1\right.$. Since

$$
\Omega_{1}\left(Z\left(O_{2}\left(N^{x}\right)\right)\right) \triangleleft N^{x}, \quad\left[P(y, x), \Omega_{1}\left(Z\left(O_{2}\left(N^{x}\right)\right)\right)\right] \subseteq \Omega_{1}\left(Z\left(O_{2}\left(N^{x}\right)\right)\right) .
$$

By Lemma 3.4, $\left[P(y, x), \Omega_{1}\left(Z\left(O_{2}\left(N^{x}\right)\right)\right)\right] \subseteq P(x, y)$. Thus, $[P(y, x)$, $\left.\Omega_{1}\left(Z\left(O_{2}\left(N^{x}\right)\right)\right)\right]=1$. But by Lemma $3.3, N_{G}(P(y, x)) \subseteq G_{y}$. Since $O_{2}\left(N^{x}\right)$ is semiregular on $X-x$, we have a contradiction to $O_{2}\left(N^{x}\right) \neq 1$. Thus, $P(x, y)$ fixes only $x$ and $y$.

Thus, $N_{N^{x}}(P(x, y))=N_{y}^{x}$. By transfer theory, $N_{y}^{x}$ is a Hall subgroup and has a normal complement $K^{x}$ in $N^{x}$. By Lemma 3.3, $N_{x}^{y}$ acts without fixed points on $K^{x}$ and is therefore cyclic. By (ii), (iii) follows.

4. $(H, K, L)$ configurations.

Definition. An $(H, K, L)$ configuration consists of groups $H, K, L$ with $H \subseteq \operatorname{Aut}(K), L \subset K$, and for all $x \in H^{\#}, C_{K}(x)=L$.

An $(H, K, L)$ configuration is constrained if $H \cong L$.

With $G$ and $N^{x}$ as in the last section, by Lemma 3.3, $N_{x}^{y}$ acts on $N^{x}$ and for all $f \in N_{x}^{y}, f \neq 1, C_{N^{x}}(f)=N_{y}^{x}$. Since $N_{y}^{x}$ and $N_{x}^{y}$ are conjugate in $G$, we have a constrained $\left(N_{x}^{y}, N^{x}, N_{y}^{x}\right)$ configuration.

LEMmA 4.1. In an $(H, K, L)$ configuration with $L \triangleleft K$, we have

(i) $[H, K] \subseteq C_{K}(L)$.

(ii) If $[H, K] \subseteq L, H$ is abelian and isomorphic to some subgroup of $L$. In particular, $|H| \leqslant|L|$. 
Proof. We use the three subgroup lemma [9, p. 19].

(i) Since $H$ centralizes $L,[H, L]=1$. Thus, $[[H, L], K]=1$. Since $L \triangleleft K,[L, K] \subseteq L$, and $[[L, K], H]=1$. Thus, $[[K, H], L]=1$ and $[K, H]$ $\subseteq C_{K}(L)$.

(ii) If $[H, K] \subseteq L$, since $[L, H]=1,[[H, K], H]=1$. Clearly, also $[[K . H], H]=1$. Thus, $[[H, H], K]=1$. Thus, $[H, H]$ centralizes $K$. Since $L \subset K$, by the definition of an $(H, K, L)$ configuration, $[H, H]=1$.

Now take $z \in K-L$. Since $[H, z] \leqslant L$, we consider the mapping $x \rightarrow$ $[x, z]$ from $H$ into $L$. In general, $[x y, z]=[x, z]^{y}[y, z]$. If $x, y \in H,[x, z]$ $\in L$ and $[x, z]^{y}=[x, z]$. Thus, $[x y, z]=[x, z][y, z]$ and the mapping $x \rightarrow$ $[x, z]$ is a homomorphism. Since $z \in K-L,[x, z]=1$ implies $x=1$, and (ii) follows.

The following construction will be frequently used. In an $(H, K, L)$ configuration take $P$ a Sylow $p$-subgroup of $H$ and $Q$ a Sylow $p$-subgroup of $K$ normalized by $P$. Set $R=Q \cap L$. Either $R=Q$, or we have a $(P, Q, R)$ configuration. Accordingly in the next several lemmas we study $(P, Q, R)$ configurations with $P, Q, R p$-subgroups for some prime $p$.

Lemma 4.2. In a $(P, Q, R)$ configuration $P$ is abelian and isomorphic to some subgroup of $R$. In particular, $|P| \leqslant|R|$.

Proof. Since $P, Q, R$ are $p$-groups, we may take some $P$-invariant subgroup $R^{\prime}$ of $Q$ such that $\left|R^{\prime}: R\right|=p$. Then we have a $\left(P, R^{\prime}, R\right)$ configuration with $R \triangleleft R^{\prime}$ and $\left[R^{\prime}, P\right] \leqslant R$. By Lemma 4.1(ii), the rest follows.

LEMMA 4.3. In a constrained $(P, Q, R)$ configuration:

(i) If $D$ is a P-invariant subgroup of $Q$, either $D \subseteq R$ or $R \subseteq D$.

(ii) $R$ is an abelian normal subgroup of $Q$.

Proof. (i) If $D$ is a $P$-invariant subgroup of $Q$, either $D \subseteq R$, or we have a $(P, D, D \cap R)$ configuration. By Lemma $4.2,|P| \leqslant|D \cap R|$. Since $|P|=|R|$ by hypothesis, $D \cap R=R$ and $R \subseteq D$.

(ii) Since $P \cong R$, by Lemma $4.2, R$ is abelian. By (i), if $C$ is characteristic in $Q, C \subseteq R$ or $R \subseteq C$. Let $Z_{i}(Q)$ be the largest member of the upper central series for $Q$ such that $Z_{i}(Q) \subseteq R$. Then $R \subseteq Z_{i+1}(Q)$. Thus, $R / Z_{i}(Q)$ $\subseteq Z\left(Q / Z_{i}(Q)\right)$ and $Z_{i}(Q) \subseteq R$, so $R \triangleleft Q$.

LEMMA 4.4. In a constrained $(P, Q, R)$ configuration:

(i) If $R \subseteq Z(Q), R$ and $P$ are elementary abelian.

(ii) If $[P, Q] \subseteq R, Q / R$ is of exponent $p$.

(iii) If $p=2$ and $[P, Q] \subseteq R$, then

(a) if $R$ is elementary abelian, $R \subseteq Z(Q)$; 
(b) if $R$ is not elementary abelian, $|Q: R|=2$, and all elements of $Q-R$ invert $R$.

PRoof. Take $z \in Q-R$ such that $|z, R\rangle: R \mid=p$ and $P$ normalizes $\langle z, R\rangle$. Then $z$ acts on $P \times R$ inducing an automorphism of the form $(x, y)$ $\rightarrow(x, \tau(x)+\varphi(y)),(x, y) \in P \times R$. Since $C_{P}(z)=1$, the mapping $x \rightarrow \tau(x)$ is an isomorphism of $P$ and $R$. Since $z$ induces a mapping of order $p, \tau(x)+$ $(\varphi \tau)(x)+\ldots+\left(\varphi^{p-1} \tau\right)(x)+\varphi^{p}(y)=y$. Since this holds for all $x$ and $y$, and since $x \rightarrow \tau(x)$ is an isomorphism, $1+\varphi+\ldots+\varphi^{p-1}=0$. If $z$ centralizes $R$, then $\varphi=1$ and so $p \cdot 1=0$ in $R$, and (i) follows.

If $p=2$, then $\varphi=-1$ and $z$ inverts $R$.

(ii) Suppose first that $[P, Q] \subseteq R$ and $R \subseteq Z(Q)$. By (i), $P$ and $R$ are elementary abelian, $Q / R$ acts on $P \times R$ (faithfully, as $C_{Q}(x)=R$, if $x \in P^{\#}$ ), and $Q / R$ centralizes $R$ and $(P \times R) / R$. Thus, all elements of $Q / R$ have quadratic minimal polynomials, and so $Q / R$ is of exponent $p$.

Now suppose only $[P, Q] \subseteq R$ and take $z \in Q-R$. Then the mapping which carries $x \in P$ into $[x, z] \in R$ is an isomorphism of $P$ and $R$. Let $B=$ $C_{R}(z)$ and take $A$ to be the pre-image of $B$ in $P$ under the mapping $x \rightarrow[x, z]$. If $x \in A,[x, z] \in R$ and $[x, z]$ commutes with $x$. Also, $[x, z] \in B=C_{R}(z)$ and $[x, z]$ commutes with $z$. Thus, $\left[x, z^{i}\right]=[x, z]^{i}[9, \mathrm{p} .19]$. Thus, $\left[x, z^{i}\right] \in B$ if $x \in A$. Since $A$ centralizes $B, A$ normalizes $\langle z, B\rangle$. Since $B$ centralizes $z,\langle B, z\rangle$ is abelian and $\langle B, z\rangle \cap R \subseteq C_{R}(z)=B$. Thus, $\langle B, z\rangle \cap R=B$. Then, we have a constrained $(A,\langle B, z\rangle, B)$ configuration with $[A,\langle B, z\rangle] \subseteq B$ and $B . \subseteq Z(\langle B, z\rangle)$. By the previous paragraph, $z^{p} \in B$. Thus, $Q / R$ is of exponent $p$, proving (ii).

(iii) If $p=2$ and $[P, Q] \subseteq R$, by (ii), $Q / R$ is of exponent 2 and so elementary abelian. By the first paragraph, every element $x$ of $Q-R$ inverts $R$. Thus, if $R$ is not elementary abelian $|Q: R|=2$. If $R$ is elementary abelian, $R \subseteq Z(Q)$.

LEMMA 4.5. In a constrained $(P, Q, R)$ configuration with $p$ odd and P cyclic, $|P|=p$.

Proof. Take a $P$-invariant subgroup $R^{\prime}$ of $Q$ such that $R \subseteq R^{\prime}$ and $\left|R^{\prime}: R\right|=p$. Since $P \cong R, R$ is cyclic. Then we have a $\left(P, R^{\prime}, R\right)$ configuration. By the classification of groups having a cyclic subgroup of index $p$ [9, p. 193], either $R^{\prime}$ is cyclic, or $\Omega_{1}\left(R^{\prime}\right)$ is elementary abelian of order $p^{2}$. Lemma 4.5 follows quickly from this.

When $p=2$, fairly precise information can be obtained.

LEMMA 4.6. In a constrained $(P, Q, R)$ configuration with $p=2$, then:

(i) If $P$ is not elementary abelian, $|Q: R|=2$ and all elementa of $Q-R$ invert $R$. 
(ii) If $|P|=2, Q$ is cyclic, dihedral, or generalized quaternion.

(iii) If $P$ is elementary abelian, but not cyclic, then either

(a) $R \subseteq Z(Q),[P, Q] \subseteq R$, and $Q / R$ is elementary abelian with $|Q / R| \leqslant|R|$, or

(b) setting $\bar{Q}=N_{Q}(P R)$, we have $|Q: \bar{Q}|=2$, and $|\bar{Q}|=|R|^{2}$.

Proof. (i) If $P$ is not elementary abelian, by Lemma 4.4(i), $C_{Q}(R) \subseteq R$, as otherwise we have a constrained $\left(P, C_{Q}(R), R\right)$ configuration. By Lemma 4.1(i), $[P, Q] \subseteq R$. By Lemma 4.4(iii), the result follows.

(ii) In the semidirect product $P Q,|P|=2$ and $C_{Q P}(P)=P R$. Thus, $P \cdot Q$ is of maximal class and contains the four-group $P \cdot R$. Thus, $P \cdot Q$ is dihedral or quasi-dihedral, and (ii) follows.

(iii) We assume $P$ is noncyclic and elementary abelain. Let $\bar{Q}=N_{Q}(P \cdot R)$. Then, $\bar{Q} / R$ is the centralizer of $P$ on $Q / R$. Clearly, $R \subset \bar{Q}$. By considering the configuration $(P, \bar{Q}, R)$, it follows from Lemma 4.4(iii), that $R \subseteq Z(\bar{Q})$, and from Lemma 4.4(ii) that $\bar{Q} / R$ is elementary abelian.

Now $\bar{Q} / R$ acts on $P \times R$ and the centralizer of any nonidentity element of $\bar{Q} / R$ on $P \times R$ is precisely $R$. Thus, we have a $(\bar{Q} / R, P \times R, R)$ configuration and, by Lemma $4.2,|\bar{Q} / R| \leqslant|R|$.

Thus, if $\bar{Q}=Q$, (iii)(a) follows. We assume $\bar{Q} \subset Q$ and show that $|Q: \bar{Q}|=$ 2 and $|\bar{Q}|=|R|^{2}$.

Take $z \in N_{P Q}(P \bar{Q})-P \bar{Q}$. As $R$ is normal in $Q$ by Lemma $4.3, z R z^{-1}=R$. Set $P^{\prime}=z P z^{-1}$. Since $P R \triangleleft P \bar{Q}$, as $\bar{Q}=N_{Q}(P \cdot R)$, also $P^{\prime} R \triangleleft P \bar{Q}$. Since $P \cdot R$ is elementary abelian, so is $P^{\prime} R$. Since $z \notin P \bar{Q}=N_{P Q}(P R), P R \neq P^{\prime} R$.

We claim that $P R \cap P^{\prime} R=R$ and $P P^{\prime} R=P \bar{Q}$.

Clearly, $P R \cap P^{\prime} R=R \cdot\left(P \cap P^{\prime} R\right)$. Since if $x \in P^{\#}, C_{P Q}(x)=P R$, and since $P \cap\left(P^{\prime} R\right)$ is central in $P^{\prime} R$ as $P^{\prime} R$ is abelian, $P \cap P^{\prime} R=1$ and $P R \cap P^{\prime} R=$ $R$. Then, since $|P|=\left|P^{\prime}\right|=|R|$,

$$
\left|P P^{\prime} R\right|=\left|P R \cdot P^{\prime} R\right|=\left|R^{3}\right| \leqslant|P \bar{Q}| \leqslant|R|^{3},
$$

and so $P P^{\prime} R=P \bar{Q}$ and $|P \bar{Q}|=|R|^{3}$. Hence, $|\bar{Q}|=|R|^{2}$.

Next we claim that all involutions of $P \bar{Q}$ lie in $P R$ or $P^{\prime} R$. Let $j$ be such an involution. Then $j=a b c, a \in P, b \in P^{\prime}, c \in R$. Since $R \subseteq Z(P \bar{Q})$ and $R$ is elementary abelian, it follows that $a b$ is an involution and so $b a=a b$. Now if $a \neq 1, C_{P Q}(a)=P R$. Since $P^{\prime} \cap(P R)=1$, it follows that $b=1$. Thus, $j=a c$ or $b c$, and the claim follows.

Since $R \subseteq Z(P \bar{Q})$ and if $a \in P^{\#}, C_{P Q}(a)=P R$, it follows that if $z \in P R-$ $R, C_{P \bar{Q}}(z)=P R$.

Thus, if $D$ is any elementary abelian subgroup of $P \bar{Q}$ and $|D|=|R|^{2}$, either $D \cap(P R) \supset R$ and $D=P R$, or $D \subseteq P^{\prime} R$ and so $D=P^{\prime} R$. Thus, $P R$ and 
$P^{\prime} R$ are the only elementary abelian subgroups of $|P \bar{Q}|$ of order $|R|^{2}$.

It follows that any element $z$ of $N_{P Q}(P \bar{Q})-P \bar{Q}$ interchanges $P R$ and $P^{\prime} R$. Thus, $\left|N_{P Q}(P \bar{Q}): P \bar{Q}\right|=2$.

We consider the action of $P$ on $Q / R$. Each element of $P$ centralizes $\bar{Q} / R$ and $|\bar{Q} / R|=|R|=|P|$. Moreover, if $x \in P,\left|C_{Q / R}(x)\right| \leqslant\left|C_{Q}(x)\right|[6$, p. 36]. Thus, if $x \in P^{\#}, C_{Q / R}(x)=\bar{Q} / R$. Setting $Q^{\prime}=Q / R$ and $R^{\prime}=\bar{Q} / R$, we have a constrained $\left(P, Q^{\prime}, R^{\prime}\right)$ configuration. Since $\left|N_{P Q}(P \bar{Q}): P \bar{Q}\right|=2,\left|N_{P Q^{\prime}}\left(P R^{\prime}\right): P R^{\prime}\right|$ $=2$. Since $P$ is elementary abelian and $|P| \geqslant 4$, it follows by induction that $N_{P Q^{\prime}}\left(P R^{\prime}\right)=P Q^{\prime}$. Thus, $|Q: \bar{Q}|=2$, proving (iii)(b).

We now return to the study of general $(H, K, L)$ configurations.

Lemma 4.7. Let $K$ be a group, $L$ a proper subgroup of $K$. Suppose for all $L_{0} \subseteq L, L_{0} \neq 1$, we have $N_{K}\left(L_{0}\right) \subseteq L$. Then, $K$ is a Frobenius group with complement $L$.

Definition. An $(H, K, L)$ configuration is minimal if all proper $H$-invariant subgroups of $K$ are contained in $L$.

LEMMA 4.8. In a minimal $(H, K, L)$ configuration $L \triangleleft K$.

Proof. If $L_{0} \subseteq L$, since $\left[H, L_{0}\right]=1, H$ normalizes $N_{K}\left(L_{0}\right)$. Since $(H, K, L)$ is minimal $N_{K}\left(L_{0}\right)=K$ or $N_{K}\left(L_{0}\right) \subseteq L$.

Let $\bar{L}$ be the largest normal subgroup of $K$ contained in $L$. Then, if $M$ is a subgroup of $L$ with $\bar{L} \subset M, N_{K}(M) \subseteq L$. By Lemma $4.7, K / \bar{L}$ is a Frobenius group with complement $L / \bar{L}$. Since $H$ normalizes the Frobenius kernel of $K / \bar{L}$, by the minimality $L / \bar{L}=1$. Thus, $L \triangleleft K$.

Proposition 4.9. In a constrained $(H, K, L)$ configuration, either

(i) $H$ is a Frobenius complement, or

(ii) $H$ is abelian, or

(iii) $H$ and $K$ are Frobenius groups and $L$ intersects the center of the Frobenius kernel of $K$ nontrivially.

Note. By (i), we mean that $H$ is a Frobenius complement in some Frobenius group, not necessarily that it acts without fixed points on $K$.

Proof. Assume neither (i) nor (ii) holds. Let $\bar{K}$ be a minimal $H$-invariant subgroup of $K$ not contained in $L$. Set $\bar{L}=L \cap K$. Then, we have a minimal $(H, \bar{K}, \bar{L})$ configuration. Thus, $\bar{L} \triangleleft \bar{K}$.

If $\bar{L}=1$, (i) holds. So assume $\bar{L} \neq 1$.

By the minimality either $C_{\bar{K}}(\bar{L}) \subseteq \bar{L}$ or $C_{\bar{K}}(\bar{L})=\bar{K}$. If $C_{\bar{K}}(\bar{L}) \subseteq \bar{L}$, by Lemma 4.1(i) and (ii), (ii) holds. So we assume $C_{\bar{K}}(\bar{L})=\bar{K}$.

We show first that $\bar{K}$ is a $p$-group.

First suppose that every prime divisor $r$ of $|H|$ also divides $|\bar{K}|$. Then also 
$r|\bar{L}|$, as the Sylow $r$-subgroup of $H$ centralizes an $r$-element of $\bar{K}$. If $r|| \bar{K}: \bar{L} \mid$, $\bar{L}$ contains a Sylow r-subgroup of $\bar{K}$. Since $\bar{L} \subseteq Z(\bar{K})$, by Burnside's transfer theorem, $\bar{K}$ has a normal $r$-complement to the Sylow $r$-subgroup of $\bar{L}$, in contradiction to the minimality of $\bar{K}$. Therefore, also $r|\bar{K}: \bar{L}|$. Thus, if $r^{\alpha}$ is the highest power of $r$ dividing $H$, it follows from Lemma 4.2 that $r^{\alpha}|\bar{L}|$. Thus, $|H||| \bar{L} \mid$. Since $\bar{L} \subseteq L \cong H, \bar{L}=L$. Since $L \subseteq Z(K), L$ and $H$ are abelian.

Thus, we may assume there is some prime $r$ dividing $|H|$ but not $|\bar{K}|$. If $x \in H,|x|=r$, since $\bar{K}$ is an $r^{\prime}$-group, $C_{\bar{K} / \bar{L}}(x)=C_{\bar{K}}(x) / L$ [9, p. 224]. Thus $C_{\bar{K} / \bar{L}}(x)=1$. By Thompson's theorem, $\bar{K} / L$ is nilpotent。 Since $\bar{L} \subseteq Z(\bar{K}), \bar{K}$ is nilpotent. By the minimality of $\bar{K}, \bar{K}$ is a $p$-group for some prime $p$.

Thus, if neither (i) nor (ii) holds, all minimal $H$-invariant subgroups of $K$ which are not contained in $L$ are groups of prime power order.

Set $\bar{K}=Q$ and $\bar{L}=R$. Then, $R \subseteq Z(Q)$ and since $H$ acts irreducibly on $Q / R, Q / R$ is elementary abelian. Let $P$ be some Sylow $p$-subgroup of $H$. By Lemma 4.2, $|P| \leqslant|R|$. Since $|R|$ divides $|L|$ and $|L|=|H|,|P|=|R|$. By Lemma 4.4(i), $P$ and $R$ are elementary abelian.

If $a \neq 1$ is a $p^{\prime}$-element of $H$ and $a$ centralizes $b \neq 1, b \in P,\langle a\rangle \times\langle b\rangle$ acts on $Q$, and $\langle a\rangle$ centralizes $C_{Q}(\langle b\rangle)=R$. By Thompson's $A \times B$ lemma [9, p. 179], $a$ centralizes $Q$, a contradiction as $C_{Q}(a)=R$. Thus for all $x \in P^{\#}, C_{H}(x)=P$.

Since any $p^{\prime}$-subgroup of $H$ acts without fixed points on $Q / R$, it follows that all $p^{\prime}$-subgroups of $H$ are Frobenius complements.

We show next that $H$ has a nontrivial abelian normal subgroup. Assume that this is not the case.

If $p \neq 2$, the Sylow 2-subgroup of $H$ is cyclic or generalized quaternion, and by the Brauer-Suzuki theorem [3] and the Feit-Thompson theorem, either $O(H) \neq 1$ or $O_{2}(H) \neq 1$. Thus, $p=2$.

Then the Sylow 2-subgroup of $H$ is elementary abelian and the centralizer of each of its involutions. By a theorem of Suzuki [15], $H=S L\left(2,2^{\alpha}\right)$ for some integer $\alpha>1$. Then $|R|=|P|=2^{\alpha}$. By Lemma 4.6(iii), $|Q / R| \leqslant 2^{\alpha+1}$. Since all elements of $H$ of odd order act without fixed points on $Q / R$, $\left(2^{\alpha}-1\right)||(Q / R)^{\#} \mid$ and $\left(2^{\alpha}+1\right)||(Q / R)^{\#} \mid$. This contradiction shows that $H$ has an abelian normal subgroup $A$.

First suppose $A$ is not a $p$-group. Since all elements of $A^{\#}$ act without fixed points on $Q / R$ as $A$ is a $p^{\prime}$-group, $A$ is cyclic. Since $P$ acts on $A$ and no $p$ and $p^{\prime}$ elements commute, $P$ is cyclic and $N_{H}(P)$ is a Frobenius group with kernel $P$. Since $\operatorname{Aut}(A)$ is abelian and $P \cap C_{H}(A)=1, N_{H}(P)=P$. By Burnside's transfer theorem, $P$ has a normal complement $N$ in $H$. As no $p$ and $p^{\prime}$ elements commute, by Thompson's theorem, $N$ is nilpotent. Since $N$ acts without fixed points on $Q / R, N$ is cyclic.

Since $R$ and $P$ are elementary abelian and $P$ is cyclic, $|R|=|P|=p$. Since 
$H$ acts irreducibly on $Q / R, Q$ is extra-special or abelian. If $Q$ is abelian, $Q=$ $C_{Q}(N) \times[Q, N][9, \mathrm{p} .177]$. Thus, $Q=R \times[Q, N]$ and $[Q, N]$ is a $P$-invariant subgroup of $Q$. So $P$ centralizes a nonidentity element of $[Q, N]$, a contradiction. Thus, $Q$ is extra-special.

Since $\left|C_{Q / R}(P)\right| \leqslant\left|C_{Q}(P)\right|=p,\left|C_{Q / R}(P)\right|=p$. Since $Q / R$ is elementary abelian, $|Q / R| \leqslant p^{p}$.

Let $Q / R=\bar{Q}=\bar{Q}_{1} \oplus \bar{Q}_{2} \oplus \cdots \oplus \bar{Q}_{k}$, where $\bar{Q}_{i}$ is the sum of equivalent minimal $N$-invariant subgroups of $\bar{Q}$ and the representations afforded by different $\bar{Q}_{i}$ and $\bar{Q}_{j}$ are distinct. Since $|\bar{Q}| \leqslant p^{p}, k \leqslant p$. If $k=p,\left|\bar{Q}_{i}\right|=p$ for all $i$. Since $N$ acts without fixed points on $\bar{Q}_{i},|N| \mid p-1$. So $P$ centralizes $N$, a contradiction. Thus, $k<p$. Since $|P|=p, P$ fixes all $\bar{Q}_{i}$. Since $C_{\bar{Q}_{i}}(P) \neq 1$ and $\left|C_{\bar{Q}}(P)\right|=p, \bar{Q}=\bar{Q}_{1}$, i.e., all minimal $N$-invariant subgroups of $\bar{Q}$ afford equivalent representations of $N$. Regarding $N$ then as a subgroup of the algebra of linear transformations on $\bar{Q}$, the subalgebra generated by $N$ is a field. Then $P$ induces an automorphism of this field of period $p$. Therefore, $|\bar{Q}|=p^{p}$. Since $Q$ is extra-special, $Q / R$ is of even dimension and so $p=2$.

Then $H \cong S_{3}$ and $Q$ is a quaternion group. Since $L \cong H, L \cong S_{3}$.

We now determine the structure of the group $K$. Let $j$ be the involution of $Q$. We first show $C_{K}(j)=Q$.

Since $H$ centralizes $j, H$ leaves $C_{K}(j)$ invariant. If 3||$C_{K}(j) \mid$, the 3-element of $H$ centralizes a 3-element of $C_{K}(j)$ and $L \subseteq C_{K}(j)$, contrary to $L \cong S_{3}$. Thus, $C_{K}(j)$ is a $3^{\prime}$-group and $N$, the 3-subgroup of $H$, acts without fixed points on $C_{K}(j) /\langle j\rangle$. Thus, $C_{K}(j) /\langle j\rangle$ is nilpotent. Thus, $C_{K}(j)$ is nilpotent. Now $H$ acts without fixed points on any $2^{\prime}$-factor of $C_{K}(j)$. But $H \cong S_{3}$ and $S_{3}$ is not a Frobenius complement. Thus, $C_{K}(j)$ is a 2-group. If $t$ is an involution of $H$, we have a constrained $\left(\langle t\rangle, C_{K}(j),\langle j\rangle\right)$ configuration. By Lemma 4.6(ii), $C_{K}(j)$ is cyclic, dihedral, or generalized quaternion. Since $C_{K}(j)$ admits the nontrivial action of a 3-element, $C_{K}(j)=Q$.

By Brauer-Suzuki [3], $K=O(K) Q$. Since $C_{K}(j)=Q, O(K)$ is abelian. Since each element of $H$ acts on $O(K)$ centralizing exactly a cyclic subgroup of order 3, $O(K)$ is a 3-group having at most three generators. Since $Q$ acts on $O(K)$ and $j$ fixes no points of $O(K), O(K)$ is homocyclic on two generators [9, p. 126]. Since the involution of $H$ has centralizer on $O(K)$ cyclic of order $3, O(K)$ $\cong Z_{3} \oplus Z_{3}$ and $K$ is the semidirect product of $Z_{3} \oplus Z_{3}$ and $Q$, so (iii) holds.

Therefore, we may suppose that if $\bar{K}$ is a minimal $H$-invariant subgroup of $K$ not contained in $L, \bar{K}$ is a $p$-group and $O_{p}(H) \neq 1$.

Thus, $H$ has an elementary abelian normal $p$-subgroup $A$. Since $C_{H}(A)=$ $P$, as $P$ is abelian and no $p$ and $p^{\prime}$ elements commute, $P \triangleleft H$. Moreover, $H$ is a Frobenius group with kernel $P$. Since there is only one prime $r$ dividing $|H|$ such that $O_{r}(H) \neq 1$, namely $r=p$, by the previous paragraph all minimal $H$ 
invariant subgroups of $K$ not contained in $L$ are $p$-groups. Thus, $p$ divides the order of any $H$-invariant subgroup of $K$ which is not contained in $L$.

Suppose $H=P S$, with $S$ a Frobenius complement of $P$. Then, as $L \cong H$, $L=R \cdot T$, with $T$ a Frobenius complement of $R$.

Take $T_{0} \subseteq T, T_{0} \neq 1$, and consider $N_{K}\left(T_{0}\right)$. If $p|| N_{K}\left(T_{0}\right) \mid$, since $N_{K}\left(T_{0}\right)$ is $H$-invariant, a $p$-element of $H$ centralizes a $p$-element of $N_{K}\left(T_{0}\right)$ and so $N_{R}\left(T_{0}\right) \neq 1$, a contradiction to the fact that $R \cdot T$ is a Frobenius group. Therefore, $N_{K}\left(T_{0}\right)$ is a $p^{\prime}$-group and $N_{K}\left(T_{0}\right) \subseteq L$.

By Lemma 4.7, $K$ is a Frobenius group with kernel $N$ and complement $T$. Since each direct factor of $N$ is $H$-invariant, $N$ is a $p$-group. By Lemma 4.3 applied to $(P, N, R), R \triangleleft N$ and so $R \cap Z(N) \neq 1$. This completes Proposition 4.9.

REMARK. It is clear from the proof of Lemma 4.9 that the statment of (iii) could be made more precise.

We determine next the structure of constrained $(H, K, L)$ configurations in which $H$ is a nonabelian Frobenius complement.

Lemma 4.10. Let $H$ be a nonabelian Frobenius complement. Let $P$ be a Sylow p-subgroup of $H$ and suppose $|P|=p$. Then either

(i) $N_{H}(P)$ is nonabelian, or

(ii) $H \cong S L(2,3) \times M$, with $p=3$, and $M$ cyclic of order prime to 2 and 3 .

PROOF. All Sylow subgroups of $H$ are cyclic or generalized quaternion. All subgroups of order $p q, p, q$ distinct primes are cyclic. Any involution lies in $Z(H)$. Thus if $p=2, N_{H}(P)=H$ is nonabelian.

Suppose $N_{H}(P)$ is abelian. By Burnside's theorem, $H$ has a normal $p$-complement $N$. If $q$ is an odd prime divisor of $N, P$ normalizes some Sylow $q$-subgroup $Q$ of $N$. Since $P$ centralizes $\Omega_{1}(Q), P$ centralizes $Q$. Since $C_{N}(P)$ is abelian, $N$ has a cyclic Hall 2 '-subgroup $M$. Also, $P$ normalizes a Sylow 2-subgroup $Q$ of $M$, and if $P$ centralizes $Q, N$, and hence $H$ is abelian. Thus $Q$ is a quaternion group, and $\left|N_{N}(M): M\right| \leqslant 4$. Also $p=3$. It follows that $M \triangleleft N$, as $(|M|, 6)=1$. From the action of $P$ on $Q, N=Q \times M$ and $H=S L(2,3) \times M$.

LEMMA 4.11. In a constrained $(H, K, L)$ configuration in which $H$ is a nonabelian Frobenius complement, $L$ is a Hall subgroup of $K$.

Proof. Suppose not and let $p$ be a prime dividing $|L|$ and $|K: L|$.

Take $P_{0}$ a subgroup of $H$ of order $p$ and $R_{0}$ a subgroup of $L$ of order $p$.

By Lemma 4.5, if $p$ is odd, $P_{0}$ and $R_{0}$ are Sylow subgroups of $H$ and $L$.

We consider $N_{K}\left(P_{0} R_{0}\right)$. Since $C_{K}\left(P_{0} R_{0}\right) \subseteq L$, and $p|| K: L \mid$, $p|| N_{K}\left(P_{0} R_{0}\right): C_{K}\left(P_{0} R_{0}\right) \mid$. Since $N_{K}\left(P_{0} R_{0}\right)$ normalizes $K \cap\left(P_{0} R_{0}\right)=R_{0}$, and $P_{0} R_{0}$ is abelian of type $(p, p), N_{K}\left(P_{0} R_{0}\right) / C_{K}\left(P_{0} R_{0}\right)$ has a unique normal sub- 
group of order $p$. Take $M$ the pre-image of this subgroup in $N_{K}\left(P_{0} R_{0}\right)$. Clearly, $|M: M \cap L|=p$ and $M \cap L \triangleleft M$.

Now $N_{H}\left(P_{0}\right)$ normalizes $P_{0}$ and $R_{0}$, and so $N_{K}\left(P_{0} R_{0}\right), C_{K}\left(P_{0} R_{0}\right)$, and $M$. If $p=2$, then $N_{H}\left(P_{0}\right)$ acts trivially on $M / M \cap L$, and we have an $\left(N_{H}\left(P_{0}\right)\right.$, $M, M \cap L)$ configuration with $\left[N_{H}\left(P_{0}\right), M\right] \subseteq M \cap L$. By Lemma 4.1, $N_{H}\left(P_{0}\right)$ is abelian. Since $P_{0} \subseteq Z(H)$, as $H$ is a Frobenius complement and $H$ is nonabelian, this is a contradiction. If $p$ is odd, set $P=P_{0}$ and $R=R_{0}$.

Again, we consider the $\left(N_{H}(P), M, M \cap L\right)$ configuration.

Suppose first that $N_{H}(P)$ does not act trivially on $M / M \cap L$. By Lemma 4.1, $C_{M}(M \cap L) \nsubseteq M \cap L$. Since $|M: M \cap L|=p, M=(M \cap L) \cdot C_{M}(M \cap L)$. Since $R \subseteq M \cap L \cap C_{M}(M \cap L)$, as $M \cap L=C_{K}(P \cdot R)$, and since $R$ is a Sylow subgroup of $M \cap L$, as $p$ is odd, it follows that $C_{M}(M \cap L)$ contains a Sylow $p$-subgroup $Q$ of $M$. Thus, $Q$ centralizes $M \cap L$ and $M=(M \cap L) \cdot Q$. Therefore, $Q \subseteq Z(M)$ and $M=Q \times O_{p^{\prime}}(M)$.

Since $Q$ char $M, N_{H}(P)$ normalizes $Q$ and centralizes $Q \cap L=R$. We obtain an $\left(N_{H}(P), Q, R\right)$ configuration. Thus, $Q$ is elementary abelian and $N_{H}(P)$ is a subgroup of the holomorph of $P$. Since $N_{H}(P)$ is a Frobenius complement, all of its subgroups of order $p q$ are cyclic, and so $N_{H}(P)=P$. But by Lemma 4.10, it follows that $H$ is abelian.

Thus, $N_{H}(P)$ acts trivially on $M / M \cap L$. By Lemma $4.1, N_{H}(P)$ is abelian. Thus, $H \cong S L(2,3) \times T$, where $T$ is cyclic. If $j$ is the central involution of $H$, then $N_{H}(P)=\langle j\rangle \times P \times T$ and $C_{K}(P R)=C_{L}(P R) \cong N_{H}(P)=\langle k\rangle \times R \times S$, with $\langle k\rangle, S \subseteq L$ and corresponding to $\langle j\rangle$ and $T$ of $H$. Again we take $Q$ a Sylow $p$-subgroup of $M$. Since $Q$ centralizes $\langle k\rangle$, it follows that $Q$ has no more than $|S|=|T|$ conjugates in $M$. Thus, some element $b \neq 1$ of $\langle j\rangle \times T$ normalizes $Q$. Then $\langle b\rangle \times P$ acts on $Q$ and the centralizer of each nonidentity element of $\langle b\rangle \times P$ is precisely $R$. By Thompson's $A \times B$ lemma, a contradiction results, and Lemma 4.11 follows.

Next we show that when $H$ is a nonabelian Frobenius complement, $L$ has a normal complement in $K$.

Lemma 4.12. In an $(H, K, L)$ configuration, suppose $N$ is an H-invariant normal subgroup of $K$ and $(|H|,|N|)=1$. Then with the natural action of $H$ on $K / N$, we have an $(H, K / N, L N / N)$ configuration.

Lemma 4.13. Suppose $K$ is a group and $L$ a Hall $\pi$-subgroup of $K$. Suppose that if $L_{0} \subseteq L, L_{0} \neq 1$, then

(i) $N_{L}\left(L_{0}\right)$ is a Hall $\pi$-subgroup of $N_{K}\left(L_{0}\right)$, and

(ii) $N_{K}\left(L_{0}\right)$ has a normal $\pi$-complement.

Then, $K$ has a normal $\pi$-complement. 
Proof. We use the following theorem of Brauer and Suzuki [2], [14]. (See also [6, p. 113].)

If $L$ is a Hall $\pi$-subgroup of $K$, then $K$ has a normal $\pi$-complement if and only if

(1) whenever two elements of $L$ are conjugate in $K$, they are conjugate in $L$;

(2) if $E$ is an elementary $\pi$-subgroup of $K$, then $E$ has some conjugate in $L$.

We verify (1). First, suppose $f_{1}$ and $f_{2}$ are two $p$-elements of $L$ which are conjugate in $K$. Clearly, we may suppose $f_{1}$ and $f_{2}$ lie in some Sylow $p$-subgroup $P$ of $K$. By Alperin's theorem [1], there is a sequence of Sylow $p$-sub-

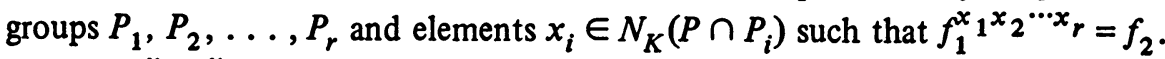
Set $g_{i}=f_{1}^{x}{ }^{\cdots} \cdots x_{i}$. Then, $g_{i}, g_{i+1} \in P \cap P_{i+1}$ and $g_{i}^{x_{i+1}}=g_{i+1}$. By hypothesis, $N_{L}\left(P \cap P_{i+1}\right)$ is a Hall $\pi$-subgroup of $N_{K}\left(P \cap P_{i+1}\right)$ and $N_{K}\left(P \cap P_{i+1}\right)$ has a normal $\pi$-complement. By the theorem of Brauer and Suzuki, there is a $y_{i+1} \in$

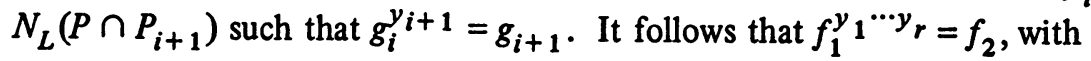
$y_{1}, \ldots, y_{r} \in L$. Thus, $f_{1}$ and $f_{2}$ are conjugate in $L$.

Suppose next that $f_{1}$ and $f_{2}$ are any two elements of $L$ conjugate in $K$. Take $g_{1} \in\left\langle f_{1}\right\rangle$ and $g_{1}$ of prime order $p$ and $g_{2}$ the conjugate of $g_{1}$ in $\left\langle f_{2}\right\rangle$. By the previous paragraph, as $g_{1}$ and $g_{2}$ are conjugate in $L$, we may assume $g_{1}=$ $g_{2}$. Then $f_{1}$ and $f_{2}$ are conjugate in $N_{K}\left(\left\langle g_{1}\right)\right)$. Since $f_{1}, f_{2} \in N_{L}\left(\left\langle g_{1}\right\rangle\right)$, as before, $f_{1}$ and $f_{2}$ are conjugate in $L$. This verifies (1).

We verify (2). If $E$ is an elementary $\pi$-group, $E$ has some cyclic normal subgroup $\langle a\rangle$. We may take $a \in L$. Then $E \subseteq N_{K}(\langle a\rangle)$. Moreover, $N_{L}(\langle a\rangle)$ is a Hall $\pi$-subgroup of $N_{K}(\langle a\rangle)$ and has a normal $\pi$-complement in $N_{K}(\langle a\rangle)$. Thus, by the theorem of Brauer and Suzuki, some conjugate of $E$ lies in $N_{L}(\langle a\rangle)$ and, hence, in $L$.

Lemma 4.13 follows.

LEMMA 4.14. In an $(H, K, L)$ configuration in which

(i) $L$ is a Hall subgroup of $K$,

(ii) $|L||| H \mid$, and

(iii) $H$ is a nonabelian group,

$L$ has a normal complement in $K$.

Proof. By induction. We let $\pi$ be the set of primes dividing $|L|$.

First we prove that if $\bar{K}$ is any $H$-invariant subgroup of $K$, then $\bar{L}=L \cap K$ is a Hall $\pi$-subgroup of $\bar{K}$. Take $P$ a Sylow $p$-subgroup of $H$ and $Q$ a Sylow $p$ subgroup of $\bar{K}$ normalized by $P$. Set $R=L \cap Q$. Then either $R=Q$, or we have a $(P, Q, R)$ configuration. By Lemma $4.2,|P| \leqslant|R|$. Since $|L||| H \mid$ and $L$ is a Hall subgroup of $K,|R| \leqslant|P|$. Thus, $R$ is a Sylow $p$-subgroup of $K$ and so $R=Q$. Thus, $\bar{L}$ is a Hall $\pi$-subgroup of $\bar{K}$.

Now if $L_{0} \subseteq L, N_{K}\left(L_{0}\right)$ is $H$-invariant, and so $N_{L}\left(L_{0}\right)$ is a Hall subgroup 
of $N_{K}\left(L_{0}\right)$. By induction either $N_{K}\left(L_{0}\right)=K$, or $N_{L}\left(L_{0}\right)$ has a normal complement in $N_{K}\left(L_{0}\right)$. Let $\bar{L}$ be the largest normal subgroup of $K$ contained in $L$. If $\bar{L} \subset L_{0} \subseteq L$, it follows that $N_{L}\left(L_{0}\right)$ is a Hall subgroup of $N_{K}\left(L_{0}\right)$ and has a normal complement in $N_{K}\left(L_{0}\right)$. By Lemma 4.13 applied to $K / \bar{L}, L / \bar{L}$ has a normal complement in $K / \bar{L}$.

Since the pre-image in $K$ of this normal complement is $H$-invariant, we may assume $\bar{L}=L$.

Next consider $C_{K}(L)$. If $C_{K}(L)=K$, as $L$ is a Hall subgroup of $K$, by Burnside's transfer theorem, $L$ has a normal complement. If $C_{K}(L) \subset K$, then $Z(L)=C_{K}(L) \cap L$ has a normal complement $N$ in $C_{K}(L)$. Then, $N$ char $C_{K}(L)$ and so $N \triangleleft K$.

If $N \neq 1$, we have an $(H, K / N, L N / N)$ configuration by Lemma 4.12. By induction on $K / N$, Lemma 4.14 follows.

So we may assume $N=1$, i.e., $C_{K}(L) \subseteq L$. But now by Lemma 4.1, $H$ is abelian, contrary to hypothesis.

Combining Lemmas 4.11 and 4.14 we have

Proposition 4.15. In a constrained $(H, K, L)$ configuration in which $H$ is a nonabelian Frobenius complement, $L$ is a Hall subgroup of $K$ and has a normal complement in $K$.

By Lemma 3.5, in the notation of $\S 3, N_{x}^{y}$ acts trivially on $N_{N}\left(N_{y}^{x}\right) / N_{y}^{x}$, i.e., $\left[N_{N} x\left(N_{y}^{x}\right), N_{x}^{y}\right] \subseteq N_{y}^{x}$. In order to gain information concerning the normalizer of $N_{y}^{x}$ in $N^{x}$, we study constrained $(H, K, L)$ configurations in which $L \triangleleft K$ and $[K, H] \subseteq L$. Already, by Lemma 4.1, $H$ and $L$ are abelian. Also, as $\left|C_{K / L}(x)\right| \leqslant\left|C_{K}(x)\right|,|K / L| \leqslant|L|$.

LEMMA 4.16. Let $\pi$ be a set of primes and $L$ an abelian normal $\pi$-subgroup of a group $K$. Suppose $B$ is a $\pi^{\prime}$-subgroup of $K$ such that $L \cdot B \triangleleft K$ and $C_{L}(B)=1$.

Let $f$ be an automorphism of $K$ which centralizes $L$ and $K / L$. Then $f$ is an inner automorphism induced by an element of $L$.

Proof. By the Schur-Zassenhaus theorem $\left[9\right.$, p. 221], $f B f^{-1}=a B a^{-1}$ for some $a \in L$. Consequently, $a^{-1} f$ normalizes $B$ and $N_{K}(B)$. Moreover, $a^{-1} f$ centralizes $L$ and $K / L$, as both $a$ and $f$ do.

By the Frattini argument and the Schur-Zassenhaus theorem, $K=L N_{K}(B)$. Moreover, $\left[a^{-1} f, N_{K}(B)\right] \subseteq L \cap N_{K}(B)=1$. Thus, $a^{-1} f$ centralizes $L$ and $N_{K}(B)$, and so $a=f$.

LEMMA 4.17. In a constrained $(H, K, L)$ configuration in which $L \triangleleft K$ and $[H, K] \subseteq L$, one of the following holds.

(i) $H, K, L$ are p-groups.

(ii) $L$ is a p-group and $K$ a Frobenius group whose kernel is a p-group. 
(iii) $K$ is a Frobenius group with kernel $L$.

(iv) For some prime $p$ dividing $L$, there is a Sylow p-subgroup $Q$ of $K$ such that $K=L \cdot Q$ and all elements of $(Q / Q \cap L)^{\#}$ act without fixed points on $O_{p},(L)$.

Proof. As remarked earlier, $H$ and $L$ are abelian. We first suppose that $H$ and $L$ are $p$-groups. We prove some preliminary statements.

(A) Any $p^{\prime}$-subgroup of $K$ acts without fixed points on $L$.

If this is not the case, there is some subgroup $T \subseteq K$ with $|T|=q, q$ a prime, $q \neq p$, centralizing $L_{0} \subseteq L$. Then in the subgroup $L T$ of $K, T$ has $\left|L: L_{0}\right|<|L|=|H|$ conjugates. Then some element $b$ of $H^{\#}$ fixes $T$. Since $b$ centralizes $K / L, b$ centralizes $T$, a contradiction.

(B) $K / L$ has no subgroup of order $p q, q$ a prime, $q \neq p$, in which the Sylow $q$-subgroup is normal.

Indeed, let $L^{\prime} \supset L,\left|L^{\prime} / L\right|=p q$, with $L^{\prime} / L q$-closed. Let $B$ be a Sylow $q$-subgroup of $L^{\prime}$. Then $C_{L}(B)=1$ by (A). Since $H$ centralizes $L$ and $K / L$, by Lemma 4.16, $H$ acts on $L^{\prime}$ as a group of inner automorphisms induced by elements of $L$. Since $|H|=|L|$, some element of $H$ centralizes the full Sylow $p$-subgroup of $L^{\prime}$, a contradiction.

Consequently:

(C) No nonidentity $p$ and $p^{\prime}$ of elements of $K$ commute.

Take $r$ the smallest prime divisor of $K / L$ other than $p$. If there is no such $r$, (i) holds.

If $r=2$, by (A) there is an involution $j \in K$ which inverts $L$. Again by (A), $C_{K}(L)$ is a $p$-group and so of odd order. Therefore, $K=C_{K}(L) C_{K}(j)$. By (C), $C_{K}(j)$ is a $p^{\prime}$-group. Again by (C), $K$ is a Frobenius group with kernel $C_{K}(L)$ and complement $C_{K}(j)$, so (ii) holds.

If $r>2$, by (A) the Sylow $r$-subgroup of $K$ is cyclic. By (B), the Sylow $r$-subgroup of $K$ is normalized by no $p$ element. Since $r$ is the smallest prime divisor of $|K / L|$ other than $p$, by Burnside's transfer theorem, $K$ has a normal $r$-complement. Repeating the process, $K$ is $p$-closed. By the Schur-Zassenhaus theorem and (C), $K$ is a Frobenius group and (ii) holds.

We now suppose $L$ is an arbitrary abelian group. We consider the action of $O_{p}(H)$ on $K / O_{p^{\prime}}(L)$. Since $O_{p}(H) \cong L / O_{p^{\prime}}(L)$, by Lemma 4.12 , we have a constrained $\left(O_{p}(H), K / O_{p^{\prime}}(L), L / O_{p^{\prime}}(L)\right)$ configuration. By the first part of this lemma, it follows that the Sylow $p$-subgroup of $K / O_{p},(L)$ is normal and that $K / O_{p^{\prime}}(L)$ is a $p$-group or a Frobenius group whose kernel is a $p$-group.

If for all $p$ which divide $|L|,(|K: L|, p)=1$, it follows that $K$ is a Frobenius group with kernel $L$, and (iii) holds.

So we may suppose that for some Sylow $p$-subgroup $Q$ of $K, Q \nsubseteq L$. 
Clearly, $Q$ may be chosen so that $O_{p}(H)$ normalizes $Q$.

We claim that all elements of $\left(Q / O_{p}(L)\right)^{\#}$ act without fixed points on $O_{p^{\prime}}(L)$. If this is not the case there is some $Q_{0}$, a subgroup of $Q$, such that $O_{p}(L) \subset Q_{0}$ and $\left|Q_{0}: O_{p}(L)\right|=p$, and $Q_{0} / O_{p}(L)$ centralizes $L_{0} \neq 1, L_{0} \subseteq O_{p^{\prime}}(L)$. $H$ normalizes $Q_{0} L$ as it acts trivially on $K / L$, and $O_{p}(H)$ normalizes $Q_{0}$, as it normalizes $Q, Q_{0} L$, and so $Q \cap\left(Q_{0} L\right)=Q_{0}$. Now $Q_{0}$ has $\left|O_{p^{\prime}}(L): L_{0}\right|$ conjugates in $Q_{0} O_{p^{\prime}}(L)$. Thus $Q_{0}$ is fixed by some $f \in O_{p},(H)^{\#}$. Thus, $\langle f\rangle \times O_{p}(H)$ fixes $Q_{0}$. By Thompson's $A \times B$ lemma, a contradiction results, and the claim follows.

Now at most one prime divides both $|L|$ and $|K: L|$. For if $p_{1}$ and $p_{2}$ divide $|L|$ and $|K: L|$, then $K / O_{p_{1}^{\prime}}(L)$ is a $p_{1}$-group or a Frobenius group whose kernel is a $p_{1}$-group. Likewise $K / O_{p_{2}^{\prime}}(L)$ is a $p_{2}$-group or a Frobenius group whose kernel is a $p_{2}$-group. Then both statements are true of $K / L$, which is ridiculous.

We now claim that $K=Q O_{p^{\prime}}(L)$, which will prove (iv). If this is not the case $K / O_{p^{\prime}}(L)$ is a Frobenius group, $Q O_{p^{\prime}}(L) \triangleleft K$, and $Q O_{p^{\prime}}(L)$ is a Hall subgroup of $K$. By the Schur-Zassenhaus theorem there is a normal complement $B$ to $Q O_{p^{\prime}}(L)$ in $K$, and $B$ may be chosen so that it normalizes $Q$. Then $Q B$ is a Frobenius group.

By Lemma 4.12, we have an $\left(O_{p^{\prime}}(H), K / O_{p}(L), L / O_{p}(L)\right)$ configuration. Since $L / O_{p}(L)$ is a Hall subgroup of $K / O_{p}(L)$, by (iii) of this lemma $K / O_{p}(L)$ is a Frobenius group with kernel $L / O_{p}(L)$. Thus, $B O_{p^{\prime}}(L)$ is a Frobenius group. It follows then that the Frobenius group $\left(Q / O_{p}(L)\right) \cdot B$ acts on $O_{p^{\prime}}(L)$ and for all $x \in\left(Q / O_{p}(L) \cdot B\right)^{\#}, C_{O_{p^{\prime}}(L)}(x)=1$. Thus, $B=1$, proving (iv).

By the remarks preceding Lemma 4.16, all the $(H, K, L)$ configurations we need consider satisfy $\left[H, N_{K}(L)\right] \subseteq L$. Moreover, as will be seen in the next section, the only cases which create difficulty occur when the Sylow 2-subgroup of $H$ is noncyclic. Accordingly, we say that an $(H, K, L)$ configuration is of type $\mathrm{A}$ if $\left[H, N_{K}(L)\right] \subseteq L$ and $H$ is an abelian group with noncyclic Sylow 2-subgroup. In the remainder of this section we study constrained $(H, K, L)$ configurations of type A. We shall show that either $L \triangleleft K$, or $K \cong S L\left(2,2^{\alpha}\right)$, with $H, L$ elementary abelian of order $2^{\alpha}$.

LEMMA 4.18. In a constrained $(H, K, L)$ configuration in which $H$ is a noncyclic abelian group, any $H$-invariant subgroup of $K$ either contains or is contained in $L$. Moreover, $N_{K}(L) \supset L$.

Proof. It suffices to show that if $\bar{K}$ is a minimal $H$-invariant subgroup of $K$ and $\bar{K} \nsubseteq L$, then $L \subseteq \bar{K}$ and $N_{\bar{K}}(L) \supset L$. Set $\bar{L}=\bar{K} \cap L$. By Lemma $4.8, \bar{L} \triangleleft \bar{K}$. Since $H$ is noncyclic $\bar{L} \neq 1$. If $C_{\bar{K}}(\bar{L}) \subseteq \bar{L}$, by Lemma 4.1, $|H| \leqslant|\bar{L}| \leqslant|L|=|H|$, and so $\bar{L}=L$ and $L \subset \bar{K}$. Moreover, $N_{\bar{K}}(L) \supset L$.

Thus, by the minimality we may assume that $C_{\bar{K}}(\bar{L}) \stackrel{\bar{K}}{=} \bar{K}$. Suppose first 
some prime $p$ divides $|H|$ but not $|\bar{K}: \bar{L}|$. If $p|| \bar{L} \mid$, as $\bar{L} \subseteq Z(\bar{K})$, by Burnside's transfer theorem, $\bar{K}$ has a normal $p$-complement, in contradiction to its minimality. So $p \nmid|\bar{L}|$. Then, by Lemma 4.16 , we have an $\left(O_{p}(H), \bar{K} / \bar{L}, 1\right)$ configuration, and so by Thompson's theorem, $\bar{K} / \bar{L}$ is nilpotent, and as $\bar{L} \subseteq Z(\bar{K}), \bar{K}$ is nilpotent. By its minimality $\bar{K}$ is a $r$-group for some prime $r$. Since $\bar{L} \neq 1$ and $|\bar{L}||| H|, r||H|$. Clearly, $r \neq 1$. Then $O_{p}(H) \times O_{r}(H)$ acts on $\bar{K}$, an $r$-group. Thus, we have a contradiction by Thompson's $A \times B$ lemma.

Thus, if $p|| H|, p||\bar{K}: \bar{L}|$. Thus, $p|| \bar{K} \mid$ and so $p|\bar{L}|$. But now, applying Lemma 4.2 to each of the Sylow subgroups of $H$, it follows that $|H||| \bar{L} \mid$, and so $\bar{L}=L$. Thus, also $N_{\bar{K}}(L) \supset L$.

In the following when an $(H, K, L)$ configuration is under consideration, $P$ will denote a Sylow 2-subgroup of $H$ and $Q$ a Sylow 2-subgroup of $K$ normalized by $P$. Then $R=Q \cap L$. As all $(H, K, L)$ configurations we consider will be constrained, by Lemmas 4.2 and $4.3, R \cong P$ and $R \triangleleft Q$.

LEMMA 4.19. In a constrained $(H, K, L)$ configuration of type A, $Q \subseteq$ $N_{K}(L)$, and

(i) if $P$ is elementary abelian, $P \subseteq Z(Q),[Q, P] \subseteq R, Q / R$ is elementary abelian, and $|Q / R| \leqslant|R|$, or

(ii) if $P$ is not elementary abelian, $|Q: R| \leqslant 2$, all elements of $Q-R$ invert $R$.

Proof. First we show that $Q \subseteq N_{K}(L)$. Set $\bar{Q}=N_{Q}(P \cdot R)$. If $R=L$, i.e., $L$ is a 2-group, since $R \triangleleft Q, Q \subseteq N_{K}(L)$. So we may suppose $O(L) \neq 1$.

Consider $N_{K}(P \cdot R)$. Now $C_{K}(P \cdot R) \triangleleft N_{K}(P \cdot R)$ and $C_{K}(P \cdot R)=L$, as $C_{K}(P)=L$ and $L$ is abelian. Thus, $N_{K}(P \cdot R) \subseteq N_{K}(L)$. Next apply Lemma 4.17(iii) and (iv). Since $O(L) \neq 1$ and $O_{2}(L) \neq 1, Q / R$ is of 2-rank at most 1 . By Lemma $4.6, \bar{Q} / R$ is elementary abelian and so $|\bar{Q}: R| \leqslant 2$. Again, by Lemma 4.6, as $R$ is noncyclic, $\bar{Q}=Q$. Thus, $Q \subseteq N_{K}(L)$.

Since $\left[H, N_{K}(L)\right] \subseteq L,[P, Q] \subseteq R$. By Lemma 4.6, the rest follows.

LEMMA 4.20. In a constrained $(H, K, L)$ configuration of type A if $L \subseteq$ $N \subseteq N_{K}(L)$ and $N \triangleleft K$, then $L \triangleleft K$.

Proof. First, suppose $O(L) \neq 1$. Since also $O_{2}(L) \neq 1$, it follows from Lemma 4.17(iii) and (iv) that $L$ is the only abelian subgroup of $N$ of order $|L|$. Thus, $L$ char $N$ and $L \triangleleft K$.

Thus, we may suppose that $L=R$, i.e., $L$ is a 2-group. Also, $H=P$. We consider two cases according as $R$ is not or $R$ is elementary abelian.

First, suppose $R$ is not elementary abelian. By Lemma 4.19, $|Q: R| \leqslant 2$. Considering the possible structures of $N_{K}(R)=N_{K}(L)$ listed in Lemma 4.17, we see that only (i) and (iii) can occur. In case (iii), $R$ char $N$ and so $R \triangleleft K$. 
In case (i), it follows that $R \subseteq N \subseteq Q$. If $N=R$, we obtained the desired conclusion, and, as $|Q: R| \leqslant 2$, we may suppose $Q=N$ and $|Q: R|=2$. Moreover, all elements of $Q-R$ invert $R$.

If $R$ is the only abelian subgroup of $Q$ of index 2, then $R$ char $Q$ and $R \triangleleft K$.

Thus we may suppose $Q$ has two abelian subgroups of index 2 . Then, some element of $Q-R$ centralizes a subgroup of index 2 in $R$. Since the elements of $Q-R$ invert $R$, it follows that $R$ is abelian of type $(4,2,2, \ldots, 2)$. Now if $Q-R$ contains an involution, all elements of $Q-R$ are involutions. Then $R$ is generated by the elements of $Q$ of order 4 , and so $R$ char $Q$ and $R \triangleleft$ $K$. Thus, we may assume all elements of $Q-R$ are of order 4. Let $\langle j\rangle=\mho_{1}(R)$ and $b \in Q-R$. Since all elements of $Q-R$ invert $R$, all elements of $Q-R$ have square $b^{2}$. If $b^{2} \neq j, b^{2}$ is the square of $|R|$ elements of $Q$ and $j$ the square of $|R| / 2$ elements of $Q$. Thus, any automorphism of $Q$ fixes $b^{2}$ and $j$, and so permutes the elements of $R-\Omega_{1}(R)$ among themselves. Since $R$ is generated by $R-\Omega_{1}(R)$, $R$ char $Q$ and $R \triangleleft K$.

Thus, we may suppose $b^{2}=j$. Then, $Q=Q_{0} \times A$, where $Q_{0}$ is a quaternion group and $A$ is elementary abelian. Now, $Q / \Omega_{1}(Q) \cong Z_{2} \times Z_{2}$ and $\Omega_{1}(Q) \triangleleft K$. Thus, $C_{K / \Omega_{1}(Q)}\left(Q / \Omega_{1}(Q)\right)=\left(Q / \Omega_{1}(Q)\right) \times M$, where $M$ is of odd order. Since $Q$ and $\Omega_{1}(Q)$ are $P$-invariant, it follows that $M$ and its pre-image in $K$ are $P$-invariant. But the pre-image of $M$ in $K$ does not contain $R$. It follows by Lemma 4.18 that $M=1$. Since $Q \triangleleft K$, it follows either that $K=Q$ and $R \triangleleft K$, or $K / \Omega_{1}(Q) \cong A_{4}$.

Suppose $K / \Omega_{1}(Q)=T \cong A_{4}$ and set $V=Q / \Omega_{1}(Q)$. Since $[K, K]$ is a $P$-invariant subgroup of $K$ and $[K, K]$ covers $V$, by Lemma $4.18, R \subseteq[K, K]$, and so $[K, K]=Q$. Thus, a 3-element of $K$ centralizes only $\langle j\rangle \subseteq \Omega_{1}(Q)$. Now $\Omega_{1}(R)=\langle j\rangle \times A$ and $A \neq 1$, as $P$ is noncyclic. Since $P$ acts trivially on $\Omega_{1}(Q)$, $P$ normalizes the action of $T / V$ on $\Omega_{1}(Q)$ and $T / V$ acts nontrivially on $\Omega_{1}(Q)$, it follows that $P$ centralizes $T / V$. Thus, $P$ induces on $T$ a 2-group of automorphisms which centralizes $T / V$. By the structure of $\operatorname{Aut}\left(A_{4}\right)$ it follows that $P$ centralizes $V, V=Q / \Omega_{1}(Q)$. It follows that $P$ leaves invariant some subgroup of $Q$ which does not contain $R$ nor is contained in $R$, a contradiction by Lemma 4.18 .

Thus, it follows that if $R$ is not elementary abelian, $R \triangleleft K$. So we may suppose next that $R$ is elementary abelian. By Lemma 4.17(i), (ii), and (iii), $N_{K}(R)$ is a 2-group or a Frobenius group whose kernel is a 2-group. Thus, $N$ $\subseteq Q$ or $Q \subseteq N$. Since we wish to show that $R \triangleleft K$, it suffices to prove this when $N=\mathrm{O}_{2}(K)$. Thus $R \subseteq N \subseteq Q$.

If $R \subseteq Q_{0} \subseteq Q$, we let $\bar{M}_{0}=O\left(N_{K}\left(Q_{0}\right) / Q_{0}\right)$ and $M_{0}$ be the pre-image of $\bar{M}_{0}$ in $N_{K}\left(Q_{0}\right)$. We first show that $M_{0} \subseteq N_{K}(R)$. Now by Lemma 4.19, $P$ 
acts trivially on $Q / R$ and so $P$ normalizes $Q_{0}$. Thus, $N_{K}\left(Q_{0}\right)$ is a $P$-invariant subgroup of $K$ and $O\left(N_{K}\left(Q_{0}\right) / Q_{0}\right)$ is a $P$-invariant subgroup of $N_{K}\left(Q_{0}\right) / Q_{0}$. Since $P$ is noncyclic, $O\left(N_{K}\left(Q_{0}\right) / Q_{0}\right)=\bar{M}_{0}=\left\langle C_{\bar{M}_{0}}(j) \mid j \in P^{\#}\right\rangle$. Let $T_{j}$ be the preimage of $C_{\bar{M}_{0}}(j)$ in $M_{0}$. Then $M_{0}=\left\langle T_{j} \mid j \in P^{\#}\right\rangle$.

If $j \in P^{\#}, T_{j}$ normalizes $Q_{0}$ and $\left\langle j, Q_{0}\right\rangle$. If $Q_{0}=R$, then $T_{j}$ normalizes $R$ and so $T_{j} \subseteq N_{K}(R)$. If $R \subset Q_{0}, Z\left(\left\langle j, Q_{0}\right\rangle\right)=R$, as $R \subseteq Z(Q)$ and $C_{Q}(j)=R$. Again, $T_{j}$ normalizes $R$ and $T_{j} \subseteq N_{K}(R)$. Thus, $M_{0}=\left\langle T_{j} \mid j \in P^{\#}\right\rangle \subseteq N_{K}(R)$, and the first claim is proved.

In particular, as $Q$ is a Sylow 2-subgroup of $K$, it follows that $N_{K}(Q) \subseteq$ $N_{K}(R)$. Since by Lemma 4.17, $Q \triangleleft N_{K}(R), N_{K}(Q)=N_{K}(R)$. Thus, if $N=Q$, the lemma follows.

Then if $N \subset Q$, by Lemma $4.19, Q / N$ is elementary abelian, and by Lemma $4.17, N_{K}(Q) / N$ is either $Q / N$ or a Frobenius group with kernel $Q / N$. If $z$ is an involution in $Q / N$ and $Q_{0}$ the pre-image of $z$ in $Q$, then the pre-image of $O\left(N_{K}\left(Q_{0}\right) / Q_{0}\right)$ in $N_{K}\left(Q_{0}\right)$ is contained in $N_{K}(R)$. Thus, $O\left(C_{K / N}(z)\right) \subseteq N_{K}(R) / N=$ $N_{K}(Q) / N$. Therefore, $O\left(C_{K / N}(z)\right)=1$.

Since $N_{K}(Q) / N$ is either $Q / N$ or a Frobenius group with kernel $Q / N$, the normalizer of $Q / N$ in $C_{K / N}(z)$ is $Q / N$. Since also $O\left(C_{K / N}(z)\right)=1, Q / N$ contains the centralizer of each of its involutions in $K / N$. By a theorem of BrauerSuzuki-Wall [4], either $Q / N \triangleleft K / N$ or $K / N \cong S L\left(2,2^{\alpha}\right)$. In the first case, $K=$ $N_{K}(Q)^{\cdot}=N_{K}(R)$ and $R \triangleleft K$.

When $K / N \cong S L\left(2,2^{\alpha}\right), P$ acts trivially on $N_{K}(Q) / N$ (as it acts trivially on $\left.N_{K}(Q) / R\right)$, and so $P$ centralizes the normalizer of a Sylow 2-subgroup of $S L\left(2,2^{\alpha}\right)$. Hence, $P$ centralizes $K / N$. Thus $P N \triangleleft P K$.

If $N=R$, of course, $R \triangleleft K$. If $R \subset N, Z(P \cdot N)=R$, and again $R \triangleleft K$. This completes the proof of Lemma 4.20.

LEMMA 4.21. In a constrained $(H, K, L)$ configuration of type A, if $|K: L|$ is odd, either $L \triangleleft K$, or $K \cong S L\left(2,2^{\alpha}\right),|H|=|L|=2^{\alpha}$.

Proof. By hypothesis, the Sylow 2-subgroup $R$ of $L$ is a Sylow 2-subgroup of $K$.

By Burnside's transfer theorem, $C_{K}(R)=R \times O\left(C_{K}(R)\right)$. Since $P \subseteq H$ is noncyclic and acts on $O\left(C_{K}(R)\right), O\left(C_{K}(R)\right) \subseteq L$. So $C_{K}(R) \subseteq L$. Since $L$ is abelian, $C_{K}(R)=L$, and as $C_{K}(R) \triangleleft N_{K}(R), N_{K}(R)=N_{K}(L)$.

By Lemma 4.17, $N_{K}(L) / O\left(N_{K}(L)\right)$ is a 2-group, or a Frobenius group whose kernel is a 2-group. Also, $N_{K}(L) / O\left(N_{K}(L)\right)=N_{K}(R) / O\left(N_{K}(R)\right)$. Thus, if $z \in R$ is an involution, the normalizer of $R$ in $C_{K}(z)$ centralizes $R$. Thus, by Burnside's transfer theorem, $C_{K}(z)=R O\left(C_{K}(z)\right)$. Then $O\left(C_{K}(z)\right)$ is an $H$-invariant subgroup of $K$, and $L \underline{\Phi} O\left(C_{K}(z)\right)$, so by Lemma 4.18, $O\left(\left(C_{K}(z)\right)\right.$ $\subseteq L$. Thus, $C_{K}(z) \subseteq L$. 
Thus, $L$ is an abelian subgroup of $K$ of even order and $L$ contains the centralizer of each of its involutions. By a theorem of Feit [7], either $L \triangleleft K$ or $K=S L\left(2,2^{\alpha}\right),|H|=|L|=2^{\alpha}$.

We now prove, by induction, that in a constrained $(H, K, L)$ configuration of type A, either $L \triangleleft K$, or $K=S L\left(2,2^{\alpha}\right),|H|=|L|=2^{\alpha}$. To simplify the statement of the lemmas, assume $K$ is a minimal counterexample. By Lemma 4.21, $R \subset Q$.

LEMMA 4.22. If $\bar{K}$ is a proper H-invariant subgroup of $K$, then $L \triangleleft \bar{K}$, if $\bar{K} \nsubseteq L$.

Proof. Already, by Lemma $4.18, L \subseteq \bar{K}$. If $L$ is not normal in $\bar{K}$, by the minimality of $K, \bar{K} \cong S L\left(2,2^{\alpha}\right),|H|=|L|=2^{\alpha}$. Then $\left|N_{\bar{K}}(L)\right|=2^{\alpha}\left(2^{\alpha}-1\right)$. Since $\left[N_{K}(L), H\right] \subseteq L,\left|N_{K}(L)\right| \leqslant|L|^{2}=2^{2 \alpha}$. Thus, $N_{\bar{K}}(L)=N_{K}(L)$. Thus, $N_{Q}(R)=R$, and $R=Q$, contrary to hypothesis.

LEMMA 4.23. Let $\bar{L}$ be the largest normal subgroup of $K$ contained in $L$. Then:

(i) $\bar{L} \subseteq Z(K)$.

(ii) $\bar{L}=1$, or $\bar{L}$ is an elementary abelian 2-group.

(iii) $K / \bar{L}$ is semisimple and $[K, K]=K$.

(iv) $\bar{L} \subset R$.

(v) If $\bar{L} \subset L_{0} \subseteq L, N_{K}\left(L_{0}\right) \subseteq N_{K}(L)$. If $L \subseteq M \subseteq N_{K}(L), N_{K}(M) \subseteq N_{K}(L)$.

Proof. (i) Clearly, $C_{K}(\bar{L})$ is an $H$-invariant subgroup of $K$. If $C_{K}(\bar{L}) \neq$ $K$, then by Lemma $4.22, C_{K}(\bar{L}) \subseteq N_{K}(L)$. Since then $L \subseteq C_{K}(\bar{L}) \subseteq N_{K}(L)$ and $C_{K}(\bar{L}) \triangleleft K$, by Lemma $4.20, L \triangleleft K$, contrary to hypothesis. Thus, $\bar{L} \subseteq Z(K)$.

(ii) Suppose $\bar{L} \neq 1$. By Lemma 4.17, $|\bar{L}|$ has at most one prime factor, as $\bar{L} \subseteq Z(K)$. Again by Lemma 4.17, if $|\bar{L}|$ is odd, $L$ contains a Sylow 2-subgroup of $K$ and $R=Q$, contrary to hypothesis. Thus, $\bar{L}$ is a 2-group. Since $\bar{L} \subseteq Z(K)$, by Lemma $4.19, \bar{L}$ is elementary abelian.

(iii) Let $C$ be a minimal characteristic subgroup not 1 of $K / \bar{L}$ and $C^{\prime}$ its pre-image in $K$. If $C^{\prime} \neq K$, by Lemma $4.22, C^{\prime} \subseteq N_{K}(L)$. Then, by Lemma 4.20, $L \triangleleft K$, contrary to hypothesis. Then $C=K / \bar{L}$ and $K / \bar{L}$ is either elementary abelian, or semisimple. If $K / \bar{L}$ is elementary abelian, since $\bar{L} \subseteq Z(K), K$ is nilpotent. Since any direct factor of $K$ is $H$-invariant, $K$ is a 2-group. Then by Lemma 4.18, $L$ and also $H$ are 2-groups. But then $L=R \triangleleft Q=K$, contrary to hypothesis. Thus, $K / \bar{L}$ is semisimple.

Then $[K, K]$ is an $H$-invariant subgroup which covers $K / \bar{L}$, and $[K, K] \nsubseteq$ $N_{K}(L)$, as $L \not K$. So $K=[K, K]$.

(iv) If $\bar{L}=R, R \triangleleft K$. We may assume $R \neq L$. Since $R=\bar{L} \subseteq Z(K)$, by Lemmas 4.17 and $4.19,|Q: R|=2$. Thus, $K / R$ has a normal 2-complement, in contradiction to (iii). 
(v) Both $N_{K}\left(L_{0}\right)$ and $N_{K}(M)$ are $H$-invariant. $N_{K}\left(L_{0}\right) \neq K$, by definition of $\bar{L} . N_{K}(M) \neq K$ by Lemma 4.20. By Lemma 4.22 the rest follows.

LEMMA 4.24. $N_{K}(Q)=Q$ if $|Q: R|=2$.

Proof. If $R=L$, by Lemma $4.17, N_{K}(R)=Q$. Then, by (v) of Lemma 4.23, $N_{K}(Q)=Q$. So we may suppose $O(L) \neq 1$. Then by Lemma $4.17, Q$ is a self-normalizing subgroup of $N_{K}(L)$.

By Lemma 4.23(iv) and (v), $N_{K}(R) \subseteq N_{K}(L)$. As $R$ char $L, N_{K}(R)=$ $N_{K}(L)$. Thus, $N_{K}(Q) \cap N_{K}(R)=N_{K}(Q) \cap N_{K}(L)=Q$.

We consider the $\left(P, N_{K}(Q), R\right)$ configuration. Since $L \cap N_{K}(Q)=R$, this is possible. Since the normalizer of $R$ in $N_{K}(Q)$ is $Q$ and $[P, Q] \subseteq R$, we have a constrained $\left(P, N_{K}(Q), R\right)$ configuration of type A. Now $N_{K}(Q) \subset K$, as $Q$ is self-normalizing in $N_{K}(L)$. So by induction $R \triangleleft N_{K}(Q)$. Thus, $N_{K}(Q) \subseteq$ $N_{K}(R)$ and $N_{K}(Q)=Q$.

LEMmA 4.25. Let $G$ be a simple group with dihedral Sylow 2-subgroup. Let $G^{*}$ be a nonsplit central extension of $G$ by an element of order 2 . Then $G^{*}$ has generalized quaternion Sylow 2-subgroup.

This can be proved directly by standard fusion arguments.

We now obtain a final contradiction to our assumption that $K$ is a minimal counterexample. Set $\bar{L}=\bar{R}$, the largest normal subgroup of $K$ contained in $L$. Then, $\bar{R} \subset R$ (Lemma 4.23(iv)). We divide the analysis into two cases.

Case A. In this case we suppose $|Q: R|=2$. By Lemma 4.17, $\left|N_{K}(L): L\right|$ $=2$. By Lemma 4.19, all elements of $Q-R$ invert $R$.

First suppose $R / \bar{R}$ is cyclic. Since $|Q: R|=2$ and all elements of $Q-R$ invert $R, Q / \bar{R}$ is dihedral or generalized quaternion. Since $K / \bar{R}$ is semisimple (Lemma 4.23(iii)), it follows from the Brauer-Suzuki theorem that $Q / \bar{R}$ is not generalized quaternion. Thus, $Q / \bar{R}$ is dihedral and, as $K / \bar{R}$ is semisimple, it is simple. Take $b \in Q-R$. Since $b$ inverts $R$, all elements of $Q-R$ have square $b^{2}$. Since $Q / \bar{R}$ is dihedral, $b^{2} \in \bar{R}$.

If $\left\langle b^{2}\right\rangle \subset \bar{R}$, we may choose $R_{0} \subset \bar{R},\left|R: R_{0}\right|=2$ and $b^{2} \in R_{0}$. Since $[K, K]=K,\left[K / R_{0}, K / R_{0}\right]=K / R_{0}$. Then $K / R_{0}$ is a central extension of $K / R$, a simple group having dihedral Sylow 2-subgroup by an element of order 2. By Lemma $4.25, Q / R_{0}$ is generalized quaternion, in contradiction to $b^{2} \in R_{0}$.

If $\left\langle b^{2}\right\rangle=\bar{R}$, since $b$ inverts $b^{2},|\bar{R}| \leqslant 2$. Then by Lemma $4.25, Q$ is generalized quarternion if $|\bar{R}|=2$, or $Q$ is dihedral if $|\bar{R}|=1$. In either case, $R$ is cyclic, contrary to hypothesis.

Thus, we may assume $R / \bar{R}$ is not cyclic.

Next we show that $R / \bar{R}$ is a T.I. set in $K / \bar{R}$. Suppose for some $x \in K$, $R \neq R^{x}$ and $R \cap R^{x} \supset \bar{R}$. Then $R \cap R^{x} \triangleleft L^{x}$, as $L^{x}$ is abelian. By Lem- 
ma 4.23(v), $L^{x} \subseteq N_{K}(L)$. Since $R^{x} \neq R, L^{x} \neq L$. Since $\left|N_{K}(L): L\right|=2$, $N_{K}(L)$ has two abelian subgroups, $L$ and $L^{x}$, of index 2. By Lemma 4.17, it follows that $L$ is abelian of type $(2,2, \ldots, 2)$ or $(4,2,2, \ldots, 2)$. Thus also, $L=R$. In the first case $Q$ is abelian, and we have a contradiction using Burnside's transfer theorem and Lemma 4.24. In the second case, $R \cap R^{x}=\Omega_{1}(R)$, as otherwise some element of $Q-R$ would centralize an element of $R$ of order 4. Thus, $Q-R$ contains an element of order 4 and so no involutions. Now $Z(Q)=\Omega_{1}(R)$ and $N(Q)$ controls the fusion of elements of $\Omega_{1}(R)$. By Lemma $4.24, N_{K}(Q)=Q$, and so no elements of $\Omega_{1}(R)$ fuse. Since $Q-R$ contains no involutions, all involutions are isolated. By Glauberman's $Z^{*}$-theorem (and as $O(K)=1$, by Lemma 4.23(ii), (iii)), $\bar{R}=\Omega_{1}(R)$. Thus, $R / \bar{R}$ is cyclic, a contradiction to what we have already proved.

Thus, if $R \neq R^{x}, R \cap R^{x}=\bar{R}$.

Suppose now for some $x \in K$ with $R \neq R^{x}$ we have $R \cap Q^{x} \supset \bar{R}$. Since $R \cap R^{x}=\bar{R}$ and $\left|Q^{x}: R^{x}\right|=2,\left|R \cap Q^{x}: \bar{R}\right|=2$. Thus, there is an element $b$ with $b^{2} \in \bar{R}$ and $b \in R \cap\left(Q^{x}-R^{x}\right)$. Since all elements of $Q^{x}-R^{x}$ invert $R^{x}$, it follows that $b$ centralizes $\Omega_{1}\left(R^{x} / \bar{R}\right)$. Let $T^{x}$ be the pre-image of $\Omega_{1}\left(R^{x} / \bar{R}\right)$ in $K$. Then $T^{x}$ normalizes $\langle\bar{R}, b\rangle$. Since $\bar{R} \subset\langle\bar{R}, b\rangle \subseteq R$, by Lemma 4.23(v), $T^{x} \subseteq N_{K}(L)$. Since $\left|N_{K}(L): L\right|=2,\left|T^{x}: T^{x} \cap L\right|=2$. Since $T^{x} \subseteq R^{x}$ and $R^{x} \cap R=\bar{R},\left|T^{x}\right| \bar{R} \mid=2$. Therefore, $\Omega_{1}\left(R^{x} / \bar{R}\right)$ is cyclic, and so $R^{x} / \bar{R}$ is cyclic, a contradiction by the first paragraph.

Thus, if $R \neq R^{x}, R \cap Q^{x}=\bar{R}$. It follows that no element of $Q / \bar{R}-R / \bar{R}$ has a conjugate in $R / \bar{R}$.

Now if $Q / \bar{R}$ is abelian, since $N_{K}(Q)=Q$ (Lemma 4.24), we have a contradiction by Burnside's transfer theorem and Lemma 4.23(iii). If $Q / \bar{R}$ is not abelian, then as all elements of $Q-R$ invert $R, Z(Q / \bar{R})=\Omega_{1}(R / \bar{R})$. Since $N_{K / \bar{R}}(Q / \bar{R})$ controls the fusion of elements of $Z(Q / \bar{R})$ and since $N_{K}(Q)=Q$, no elements of $Z(Q / \bar{R})$ fuse. Thus, by the previous paragraph, all involutions of $Z(Q / \bar{R})$ are isolated. By Glauberman's theorem, we get a final contradiction.

Case B. In this case, we suppose $|Q: R| \geqslant 4$. By Lemma 4.19, $R$ and $Q / R$ are elementary abelian and $R \subseteq Z(Q)$. By Lemma 4.17, $L=R$. By Lemma 4.23(v), $N_{K}(Q) \subseteq N_{K}(R)$ and by Lemma 4.17, $N_{K}(Q)=N_{K}(R)$.

First, we suppose that $N_{K}(Q)=Q$. Since $N(Q)$ controls the fusion of elements of $Z(Q)$, no elements of $Z(Q)$ are fused. Thus, either all elements of $R$ are isolated, or some involution $t \in Q-R$ fuses into $R$. If all elements of $R$ are isolated, by Glauberman's $Z^{*}$-theorem, $R \triangleleft K$, contrary to hypothesis. Thus, some $t \in Q-R$ fuses into $R$. Then, $t \in R^{x}$ for some $x \in K$ and $R^{x} \neq$ $R$ as $t \notin R$. Since $t \notin R, t \notin R$. By Lemma 4.23(v), $N_{K}\left(\left\langle t^{x^{-1}}, \bar{R}\right\rangle\right) \subseteq Q$. Thus, $N_{K}(\langle t, \bar{R}\rangle) \subseteq Q^{x}$. Since $R \subseteq Z(Q)$ and $t \in Q, t$ centralizes $R$. Thus, $R$ normalizes $\langle t, \bar{R}\rangle$ and $R \subseteq Q^{x}$. Since $Q^{x} / R^{x}$ is elementary abelian, $R R^{x} \triangleleft Q^{x}$. 
Also, $R$ centralizes $R^{x}$ as $R^{x} \subseteq Z\left(Q^{x}\right)$. Since $Q=N_{K}(R), R R^{x} \subseteq Q$. Then $R \subseteq$ $R R^{x} \subseteq Q$ and by Lemma $4.23(\mathrm{v}), Q^{x} \subseteq Q$. Thus, $Q^{x}=Q$ and $R^{x}=R$, contrary to hypothesis.

Next, suppose $N_{K}(Q) \supset Q$. By Lemma $4.17, N_{K}(Q)$ is a Frobenius group. Since $\bar{R} \subseteq Z(K) . \bar{R}=1$.

If for some $x \in K, R \cap R^{x} \neq 1$, then $R \cap R^{x} \subseteq Z\left(Q^{x}\right)$, as $R^{x} \subseteq Z\left(Q^{x}\right)$. Thus, $R \cap R^{x} \triangleleft Q^{x}$. By Lemma 4.23(v), $N_{K}\left(R \cap R^{x}\right) \subseteq N_{K}(R)$ and $N_{K}(R)$ is a Frobenius group with kernel $Q$. Thus, $Q^{x} \subseteq Q$. Since $N_{K}(Q)=N_{K}(R), R^{x}=$ $R$. Thus, $R \cap R^{x}=1$ if $R \neq R^{x}$.

Next suppose for some $x \in K, R \cap Q^{x} \neq 1$. Take $j \in R \cap Q^{x}, j \neq 1$. Then $j$ centralizes $R^{x}$. By Lemma 4.23(v), $C_{K}(j) \subseteq N_{K}(R)$. Thus, $R^{x} \subseteq N_{K}(R)$, and as $N_{K}(R)$ is a Frobenius group with kernel $Q, R^{x} \subseteq Q$. Since $R \cap R^{x}=1$, if $R \neq R^{x}$, and $|Q| \leqslant|R|^{2}, R R^{x}=Q$. Since also $\left[N_{K}(R), P\right] \subseteq R,\left|N_{K}(R)\right| \leqslant$ $|R|^{2}$. Thus, $Q=N_{K}(R)$, contrary to hypothesis. Thus, if $R \cap Q^{x} \neq 1, R=R^{x}$ and $Q=Q^{x}$.

Thus, if $z$ is some involution of $Q-R, z$ is not fused into $R$. We study $C_{K}(z)$.

Since $R \subseteq Z(Q), R \subseteq C_{K}(z)$. Take $T$ a Sylow 2-subgroup of $C_{K}(z)$ containing $\langle R, z\rangle$. Then $T$ is contained in some Sylow 2-subgroup $Q^{x}$ of $K$. Since $R \subseteq Q^{x}, Q^{x}=Q$. Thus, $T \subseteq Q$. Since $R \subseteq T \subseteq Q$, by Lemma 4.23(v), $N_{K}(T)$ $\subseteq N_{K}(Q)$.

Thus, since $N_{K}(Q)$ is Frobenius group with kernel $Q, N_{C_{K}(z)}(T)=T$. Now $R \subseteq Z(T)$ and $N_{C_{K}(z)}(T)$ controls the fusion of involutions of $Z(T)$ in $C_{K}(z)$. Since no involutions of $R$ fuse into $Q-R$ (and hence $T-R$ ), all involutions of $R$ are isolated in $C_{K}(z)$. Since $R$ is noncyclic, $O\left(C_{K}(z)\right) \subseteq\left\langle C_{K}(j) \mid j \in R^{\#}\right\rangle$. By Lemma 4.23(v), $C_{K}(j) \subseteq Q$, if $j \in R^{\#}$. Hence, $O\left(C_{K}(z)\right)=1$. By Glauberman's $Z^{*}$-theorem, $R \triangleleft C_{K}(z)$. Thus, $C_{K}(z) \subseteq N_{K}(R)$ and since $N_{K}(R)$ is a Frobenius group, $C_{K}(z) \subseteq Q$.

It follows that $N_{K}(Q)$ is a strongly embedded subgroup of $K$. By a standard argument (see, for example, [9, p. 306]), $K$ has a single class of involutions. Since $N_{K}(Q)$ controls the fusion of elements of $R, N_{K}(Q)$ is transitive on the elements of $R^{\#}$. Thus $\left|N_{K}(Q): Q\right| \geqslant|R|-1$. Since $\left[N_{K}(R), P\right] \subseteq R, \mid N_{K}(R)$ : $R|\leqslant| R \mid$. Thus, $R=Q$, contrary to hypothesis.

Thus, we have proved

Proposition 4.26. In a constrained $(H, K, L)$ configuration in which

(i) $H$ is an abelian group whose Sylow 2-subgroup is noncyclic,

(ii) $\left[H, N_{K}(L)\right] \subseteq L$, then either $L \triangleleft K$, or $K \cong S L\left(2,2^{\alpha}\right)$ and $|H|=|L|=2^{\alpha}$.

5. Completion of the proof of Theorem A. In this section, $G$ is a doublytransitive group on $X, x \in X, N^{x} \triangleleft G_{x}$. As usual, $N^{y}$ is the unique conjugate 
of $N^{x}$ which lies in $G_{y}$. We assume $N^{x} \cap N^{y}=1$ if $x \neq y$, and $N_{y}^{x} \neq 1$, if $y \in X-x$. We complete the proof of Theorem A.

By Lemma 3.3, we have a constrained $\left(N_{x}^{y}, N^{x}, N_{y}^{x}\right)$ configuration. By Propositions 4.9 and 4.15 , we have either

(1) $N^{x}$ is a Frobenius group with kernel $K^{x}$ and $Z\left(K^{x}\right) \cap N_{y}^{x} \neq 1$, or

(2) $N_{y}^{x}$ is a nonabelian Frobenius complement and a Hall subgroup of $N^{x}$. Moreover, $N_{y}^{x}$ has a normal complement $K^{x}$ in $N^{x}$, or

(3) $N_{y}^{x}$ is abelian.

In both (1) and (2), $K^{x}$ char $N^{x}$ and $K^{x} \triangleleft G_{x}$. In (1), by Lemma 3.19(i), Theorem A follows. In (2), by taking $M^{x}$ a minimal characteristic subgroup of $N^{x}$ which properly contains $K^{x}$, the hypotheses of Lemma 3.19(ii) are satisfied and Theorem A follows. Thus, in the remainder of this section we confine our attention to the case in which $N_{y}^{x}$ is abelian. Also, as we may replace $N^{x}$ by any characteristic subgroup $K^{x}$ with $K_{y}^{x} \neq 1$, we may assume that any proper characteristic subgroup of $N^{x}$ in $N^{x}$ is semiregular on $X-x$.

LEMma 5.1. If $N^{x}$ has a nonidentity abelian normal subgroup, Theorem A follows.

Proof. We may take $A^{x} \neq 1$ an abelian characteristic $p$-subgroup of $N^{x}$, and $A^{x}$ semiregular on $X-x$.

We first claim that if $f \in N_{x}^{y}, x \neq y, f \neq 1$, then $C_{A} x(f)=1$. Indeed, by Lemma 3.1, $C_{G}(f) \subseteq G_{y}$. Thus, $C_{A}(f) \subseteq A_{y}^{x}=1$.

Thus, it follows that $N_{x}^{y}$ is cyclic and $p \nmid\left|N_{x}^{y}\right|$.

Next, suppose $B \subseteq N_{y}^{x}, B \neq 1$. We claim: $C_{A}(B)=1$. Clearly, it suffices to prove this when $B$ is an $r$-group for some prime $r$. Since $N_{y}^{x}$ is cyclic, by Lemma 3.6, $N_{N^{x}}(B)=N_{N^{x}}\left(N_{y}^{x}\right)$. Thus,

$$
C_{A^{x}}(B)=N_{N^{x}}(B) \cap A^{x}=N_{N^{x}}\left(N_{y}^{x}\right) \cap A^{x}=C_{A^{x}}\left(N_{y}^{x}\right) .
$$

Thus, it suffices to prove $C_{A^{x}}\left(N_{y}^{x}\right)=1$.

Since $N_{x}^{y}$ acts on $N^{x}$ and centralizes $N_{y}^{x}, N_{x}^{y}$ normalizes $N_{N^{x}}\left(N_{y}^{x}\right)$ and $C_{A} x\left(N_{y}^{x}\right)$. Thus, $\left[N_{x}^{y}, C_{A}\left(N_{y}^{x}\right)\right] \subseteq A^{x}$. On the other hand, by Lemma 3.5, $\left[N_{x}^{y}, N_{N^{x}}\left(N_{y}^{x}\right)\right] \subseteq N_{y}^{x}$. Thus, $\left[N_{x}^{y}, C_{A}\left(N_{y}^{x}\right)\right] \subseteq A^{x} \cap N_{y}^{x}=1$. follows.

Since $C_{A^{x}}\left(N_{x}^{y}\right)=1$, it follows that $C_{A^{x}}\left(N_{y}^{x}\right)=1$, and the second claim

Next we show that $N_{y}^{x}$ is a Hall subgroup of $N^{x}$. Let $P$ be a Sylow $r$-subgroup of $N_{x}^{y}, R$ a Sylow $r$-subgroup of $N_{y}^{x}$ and $Q$ a Sylow $r$-subgroup of $N^{x}$ normalized by $P$ and containing $R$. Assume $Q \supset R$.

Then $N_{P Q}\left(\Omega_{1}(P) \times \Omega_{1}(R)\right) \supset P \cdot R$. Since $C_{P Q}\left(\Omega_{1}(P) \times \Omega_{1}(R)\right)=P \cdot R$ and since $\Omega_{1}(R) \triangleleft N_{P Q}\left(\Omega_{1}(P) \times \Omega_{1}(R)\right)$, it follows that all cyclic subgroups of $\Omega_{1}(P) \times \Omega_{1}(R)-\Omega_{1}(R)$ are conjugate. Since $\Omega_{1}(P)$ and $\Omega_{1}(R)$ are conjugate, 
all cyclic subgroups of $\Omega_{1}(P) \times \Omega_{1}(R)$ are conjugate.

But we have seen that no nonidentity elements of $N_{y}^{x}$ and $N_{x}^{y}, y \in X-x$, centralize nonidentity elements of $A^{x}$. Hence, $\Omega_{1}(P) \times \Omega_{1}(R)$ acts on $A^{x}$ and no element of $\left(\Omega_{1}(P) \times \Omega_{1}(R)\right)^{\#}$ centralize elements of $\left(A^{x}\right)^{\#}$, a contradiction.

Thus, $N_{y}^{x}$ is a Hall subgroup of $N^{x}$.

Now if $N_{y}^{x}$ is of odd order, Theorem A follows by Lemma 3.19(iii). If $\left|N_{y}^{x}\right|$ is even, as $N_{y}^{x}$ is cyclic and contains a Sylow 2-subgroup of $N^{x}$, the Sylow 2-subgroup of $N^{x}$ is cyclic. Thus, by Burnside's transfer theorem and the Feit-Thompson theorem, $N^{x}$ is solvable.

Let $\pi$ be the set of primes dividing $\left|N_{y}^{x}\right|$. Then, $O_{\pi^{\prime}}\left(N^{x}\right) \neq 1$ and as $O_{\pi^{\prime}, \pi}\left(N^{x}\right) \cap N_{y}^{x} \neq 1, O_{\pi^{\prime} \pi}\left(N^{x}\right)=N^{x}$. Thus, $N^{x}=O_{\pi^{\prime}}\left(N^{x}\right) N_{y}^{x}$.

By the minimality of $N^{x}, N_{y}^{x}$ is cyclic of prime order, and the hypotheses of Lemma 3.19(ii) hold. Thus, Theorem A follows.

LEMma 5.2. If $\left|N_{y}^{x}\right|$ is odd, Theorem A holds.

This follows from Lemma 3.19(iii).

In the remainder we may assume $N_{y}^{x}$ is abelian of even order. By Lemma 5.1, either Theorem A holds, or $O\left(N^{x}\right)=1$ and $O_{2}\left(N^{x}\right)=1$. We assume $O\left(N^{x}\right)=1$ and $O_{2}\left(N^{x}\right)=1$ and derive a contradiction.

Take $K^{x}$ a minimal characteristic subgroup of $N^{x}$. Then $K^{x}$ is of even order and if $K_{y}^{x}=1$, by Theorem C(ii), $O_{2}\left(K^{x}\right) \neq 1$, and so $O_{2}\left(N^{x}\right) \neq 1$. Thus, $K_{y}^{x} \neq 1$. Thus, by the minimal choice of $N^{x}, N^{x}=K^{x}$, and $N^{x}$ is semisimple.

LEMma 5.3. The Sylow 2-subgroup of $N_{y}^{x}$ is not cyclic.

Proof. Suppose the Sylow 2-subgroup of $N_{y}^{x}$ is cyclic. Let $T(x, y)$ be the unique subgroup of $N_{y}^{x}$ of order two. By Lemma 1.8, the family of sets $\left\{\Gamma_{T}(x, y)\right\}$ (with $\Gamma_{T}(x, y)=F_{T(x, y)}$ ) forms a block design on $X$ preserved by $G$. We denote this block design by $B$.

If $y \in X-x$, let $P$ be a Sylow 2-subgroup of $N_{x}^{y}, R$ a Sylow 2-subgroup of $N_{y}^{x}$ and $Q$ a Sylow 2-subgroup of $N^{x}$ containing $R$ and normalized by $P$. Then, $Q \supset R$, as $N^{x}$ is semisimple. So we have a $(P, Q, R)$ configuration. By Lemma 4.6(i) and (ii), $Q$ is cyclic, dihedral, quasi-dihedral, or generalized quaternion. Since $N^{x}$ is semisimple, $Q$ is dihedral or quasi-dihedral and $N^{x}$ is simple.

From the structure of $Q, N_{Q}(T(x, y) T(y, x)) \supset N_{P}(T(x, y) T(y, x))$, and so all involutions of $T(x, y) T(y, x)-T(x, y)$ are conjugate. Since $T(x, y)$ and $T(y, x)$ are conjugate, all involutions of $T(x, y) T(y, x)$ are conjugate in $G$, and each has fixed point set $\Gamma_{T}(x, y)$.

By the structure of the Sylow 2-subgroups of $N^{x}, N^{x}$ has one class of involutions. Thus, all involutions of $N^{x}$ fix at least two points.

Next, we note that if $j \in N^{x}$ is an involution, $X$ is the union of blocks 
of $B$ fixed by $j$. Clearly, $x$ belongs to such a block. If $y \neq x$ is fixed by $j, y \in$ $B(x, y)$, and $B(x, y)$ is fixed by $j$. If $y$ is not fixed by $j$, then $y \in B(y, j(y))$, and $B(y, j(y))$ is fixed by $j$.

We claim: If $j \in N^{x}$ is an involution, then $j$ fixes all blocks which contain $x$. By the previous paragraph, it suffices to show that any block fixed by $j$ contains $x$.

Let $B$ be a block fixed by $j$. If $j$ fixes $a, b \in B, j$ normalizes $T(a, b)$, and as $|T(a, b)|=2, j$ centralizes $T(a, b)$. Since $j \in N^{x}$, by Lemma 3.1, $T(a, b)$ fixes $x$. Since $B=F_{T(a, b)}, x \in B$. If $j$ interchanges $a, b \in B, j$ normalizes $T(a, b) T(b, a)$. Since $T(a, b) T(b, a) \cong Z_{2} \times Z_{2}, j$ centralizes an involution $z$ of $T(a, b) T(b, a)$. By Lemma 3.1, $z$ fixes $x$. Since $B$ is the fixed point set of $z, x \in B$.

So every involution of $N^{x}$ fixes all blocks which contain $x$, By the simplicity of $N^{x}, N^{x}$ fixes all blocks which contain $x$.

But now if $B=\Gamma_{T}(x, y), N^{x}$ fixes the set $B$ and $T(x, y) \subseteq N^{x}$ fixes all points of $B$. Since $N^{x}$ is simple, $N^{x}$ fixes all points of $B$, and so at least two points, a contradiction.

Thus, $N_{y}^{x}$ has noncyclic Sylow 2-subgroup. By Proposition 4.26, either $N_{y}^{x} \triangleleft N^{x}$, or $N^{x}=S L\left(2,2^{\alpha}\right)$, and $\left|N_{y}^{x}\right|=\left|N_{x}^{y}\right|=2^{\alpha}$. Since $N^{x}$ is semisimple, $N^{x}=S L\left(2,2^{\alpha}\right)$.

We let $B$ be the block design $\left\{\Gamma_{N}(u, v)\right\}$. Since $N_{y}^{x}$ is a Sylow 2-subgroup of $N^{x}$, by the Frattini argument, $G_{x}=N_{G_{x}}\left(N_{y}^{x}\right) N^{x}$. If $B=\Gamma_{N}(x, y), N_{G_{x}}\left(N_{y}^{x}\right)$ fixes $B$. It follows that $N^{x}$ is transitive on the blocks which contain $x$. Now the subgroup of $N^{x}$ fixing $B$ is $N_{N^{x}}\left(N_{y}^{x}\right)$. Thus, there are exactly $1+2^{\alpha}$ blocks of $B$ containing $x$.

Suppose $|B|=1+l$. By Lemma 1.1 (iii), $1+l \leqslant 1+2^{\alpha}$, and so $l \leqslant 2^{\alpha}$. On the other hand, $\left|N_{G_{x}}\left(N_{y}^{x}\right) / N_{y}^{x}\right|=2^{\alpha}-1$ and $N_{G_{x}}\left(N_{y}^{x}\right) / N_{y}^{x}$ acts without fixed points on $B-x$. Thus, $\left(2^{\alpha}-1\right)$ divides $l$. It follows that $l=2^{\alpha}-1$.

Thus, $|X|=1+\left(2^{\alpha}-1\right)\left(2^{\alpha}+1\right)=2^{2 \alpha}$ and $|B|=2^{\alpha}$. It follows that $(X, B)$ is an affine plane with 2-transitive automorphism group. Hence, $G$ has a regular normal subgroup by [12]. By Lemma 3.7, we obtain a contradiction, and hence Theorem $\mathbf{A}$.

\section{REFERENCES}

1. J. L. Alperin, Sylow intersections and fusion, J. Algebra 6 (1967), 222-241. MR 35 \#6748.

2. R. Brauer, On quotient groups of finite groups, Math. Z. 83 (1964), 72-84. MR 28 \#3088.

3. R. Brauer and M. Suzuki, On finite groups of even order whose 2-Sylow group is a quaternion group, Proc. Nat. Acad. Sci. U.S.A. 45 (1959), 1757-1759. MR 22 \#31.

4. R. Brauer, M. Suzuki and G. E. Wall, A characterization of the one-dimensional unimodular projective groups over finite fields, Illinois J. Math. 2 (1958), 718-745. MR 21 \#3487. 
5. W. Burnside, Theory of groups, 2nd ed., Dover, New York, 1967.

6. W. Feit, Characters of finite groups, Benjamin, New York, 1967. MR 36 \#2715.

7. A characterization of the simple groups $\operatorname{SL}\left(2,2^{\alpha}\right)$, Amer. J. Math. 82

(1960), 281-300. MR 22 \#4785.

8. G. Glauberman, Central elements in core-free groups, J. Algebra 4 (1966), 403-420. MR 34 \#2681.

9. D. Gorenstein, Finite groups, Harper \& Row, New York and London, 1968. MR 38 \#229.

10. C. Hering, W. M. Kantor and G. M. Seitz, Finite groups with a split BN-pair of rank 1. I, J. Algebra 20 (1972), 435-475. MR 46 \#243.

11. M. O'Nan, $A$ characterization of $L_{n}(q)$ as a permutation group, Math. Z. 127 (1972), 301-314. MR 47 \#310.

12. T. G. Ostrom and A. Wagner, On projective and affine planes with transitive collineation groups, Math. Z. 71 (1959), 186-199. MR 22 \#1843.

13. E. Shult, On a class of doubly-transitive groups, Illinois J. Math. 16 (1972), 434-445. MR 45 \#5211.

14. M. Suzuki, On the existence of a Hall normal subgroup, J. Math. Soc. Japan 15 (1963), 387-391. MR 28 \#2157.

15. - Finite groups of even order in which Sylow 2-groups are independent, Ann. of Math. (2) 80 (1964), 58-77. MR 29 \#145.

16. H. Wielandt, Finite permutation groups, Lectures, University of Tübingen, 1954/55; English transl., Academic Press, New York, 1964. MR 32 \#1252.

17. - Über die Existenz von Normalteilen in endlichen Gruppen, Math. Nachr. 18 (1958), 274-280. MR 21 \#2009.

18. E. Witt, Die 5-fach transitiven Gruppen von Mathieu, Abh. Math. Sem. Univ. Hamburg 12 (1937), 256-264.

19. C. Hering, On subgroups with trivial normalizer intersection, J. Algebra 20 (1972), 622-629. MR 48 \#385.

20. E. Shult, On the fusion of an involution in its centralizer (unpublished).

DEPARTMENT OF MATHEMATICS, RUTGERS UNIVERSITY, NEW BRUNSWICK, NEW JERSEY 08903 ILLINOIS 60637

DEPARTMENT OF MATHEMATICS, UNIVERSITY OF CHICAGO, CHICAGO, 